\title{
The novel VIKOR methods for generalized Pythagorean fuzzy soft sets and its application to children of early childhood in COVID-19 quarantine
}

\author{
Murat Kirişci ${ }^{1}$ (D) $\cdot$ Ibrahim Demir $^{2}$ (D) $\cdot$ Necip Şimşek ${ }^{3} \cdot$ Nihat Topaç $^{4} \cdot$ Musa Bardak $^{5}$
}

Received: 2 March 2021 / Accepted: 17 August 2021 / Published online: 15 September 2021

(C) The Author(s), under exclusive licence to Springer-Verlag London Ltd., part of Springer Nature 2021

\begin{abstract}
In this work, the new VIKOR methods are established using the generalized Pythagorean fuzzy soft sets (GPFSSs). For GPFSSs, the distance measures such as Hamming, Euclidean, and generalized are given. Further, the basic characteristics of these distance measures are examined. Fuzzy and soft sets are strong instruments for uncertainty. This strongness has been demonstrated by the GPFSS combining Pythagorean fuzzy sets and soft sets and applied to imprecise and ambiguous information. In this context, new remoteness index-based methods have been proposed, which are dissimilar from available VIKOR methods. The displaced and fixed ideals positive and negative Pythagorean fuzzy values (PFV) were defined. Thus, based on this definition, displaced positive ideal remoteness indices, negative ideal remoteness indices, and fixed positive ideal, negative ideal remoteness indices were discussed. Two different weights are used here: weights based on OF preference information and precise weights calculated with the expectation score function. The VIKOR method given here provides a different way from canonical VIKOR methods: rank candidate alternatives and determining a compromise solution based on different preference structures. The processes principles of the newly defined GPFSSs VIKOR methods are given by four algorithms. An example of these algorithms is given with the behavioral development and cognitive development of the children of Early Childhood children in the COVID-19 quarantine.
\end{abstract}

Keywords Remoteness Index-Based VIKOR method · Generalized Pythagorean fuzzy soft set $\cdot$ Multi-attribute decision making $\cdot$ Expectation score function $\cdot$ Early childhood

Murat Kirișci

murat.kirisci@istanbul.edu.tr

1 Department of Biostatistics, Cerrahpasa Faculty of Medicine, Istanbul University-Cerrahpasa, Istanbul, Turkey

2 Department of Statistics, Yildiz Technical University, Istanbul, Turkey

3 Department of Mathematics, Istanbul Commerce University, Istanbul, Turkey

4 Department of Early Childhood Educaiton, Istanbul University-Cerrahpasa, Istanbul, Turkey

5 Department of Early Childhood Educaiton, Istanbul Sabahattin Zaim University, Istanbul, Turkey

\section{Introduction}

Uncertainty is a crucial concept for decision-making problems. It is not easy to make precise decisions in life since each information contains vagueness, uncertainty, imprecision. Fuzzy Set(FS) Theory, Zadeh's [49] pioneering work, proposed a membership function to solve problems such as vagueness, uncertainty, imprecision, and this function took value in the range of $[0,1]$. FS Theory had solved many problems in practice, but there was no membership function in real life, which only includes acceptances. Rejection is as important as acceptance in real life. Atanassov [3] clarified this problem and posed the Intuitionistic Fuzzy Set(IFS) Theory using the membership function as well as the non-membership function. In IFS, the sum of membership and non-membership grades is 1 . This condition is also a limitation for solutions of vagueness, uncertainty, imprecision. Yager [46] has presented a 
solution to this situation and suggested Pythagorean Fuzzy Sets(PFS). PFS is more comprehensive than IFS because it uses the condition that the sum of the squares of membership and non-membership grades is equal to or less than 1. PFS is also a particular case of the Neutrosophic Set initiated by Smarandache [43]. There are many studies in the literature on FS, IFS, and PFS theories $[1,2,13-20,23-27,29,30,38-41,43-45,47,48,51]$. Despite all the possible solutions, these theories have limitations. How to set the membership function in each particular object and the deficiencies in considering the parametrization tool can be given as examples of these limitations. These limitations handicap decision-makers from making a correct decision during the analysis.

A new method, called Soft Set, was proposed by Molodtsov [33], in which the preferences for each alternative were given in distinct parameters, and thus a solution was found for the limitations expressed. Immediately after the occurrence of SS theory, Fuzzy Soft Sets [29] and Intuitionistic Fuzzy Soft Set(IFSS) [30] were defined and their various properties were studied [31, 32]. Pythagorean Fuzzy Soft Set(PFSS) is defined by Peng et al. [38]. PFSS is a natural generalization of IFSS and is a parameterized family of PFSs. In [4, 5, 22, 34, 42], the main features of PFSS were examined and applied to various areas such as medical diagnosis, selection of a team of workers for business, stock exchange investment problem. The benefit of these extended theories is that they are capable of simplifying the characterization of real-life cases with the help of their parameterized feature.

It is possible that people may hesitate during decisionmaking. In order to avoid human hesitations from adversely affecting the decision-making process, hesitation value is also taken into account in PFS, just like IFS. Thus, experts may have hesitations about membership grades. If the expert participating in the decision process is only one person, this expert's error or bias will affect the process negatively. However, hesitation is subjective and the expert's hesitation can be directed by her/his own perceptions. In this case, enriching the decision process, making the evaluation with alternative decisions more meaningful, combining the subjective evaluations of more than one expert instead of the subjective evaluation of a single expert will provide a healthier decision-making process. With this in mind, Agarwal et al. [1] defined Generalized Intuitionistic Fuzzy Soft Sets(GIFSS). Feng et al. [14] identified some problems and difficulties in the definition of GIFSS and operations related to GIFSS in the manuscript of Agarwal et al [1]. Kirisci [27] defined GPFSS, considering the fixes in [14].

GPFSS ensures the frame for evaluating the reliability of the info in the PFSS so as to compensate for any distortion in the info given. The most important benefit of incorporation of the generalized parameter into the analysis is to decrease the likelihood of errors induced by the imprecise info by taking the chairperson's view on the same. For example, a patient may give wrong information to a physician about her/his symptoms. If the physician does not notice this wrong information, errors in diagnosis and treatment will occur. In this case, an experienced physician can measure the reliability of the information given by the patient with a generalization parameter. So, there is a requirement for a generalization parameter, demonstrating an expert's level of confidence in the reliability of the info, respectably making the approach quite close to real-world cases. This assists in extracting the singular bias from the input data and gets more credibility to the final decision. GPFSS has a generalization parameter to states the uncertainties.

Usually, the most present multiple criteria decision analysis (MCDA) methods goal to discover a unique solution that maximum achieves the general criteria as much as possible. The VIKOR method is skilled in specifying a set of compromise solutions(CS) in the existence of contradictory criteria according to the choices of the decision-makers. The VIKOR method developed by Opricovic and Tzeng $[35,36]$ focuses on ranking and selecting from a set of alternatives, and determines compromise solutions for a problem with conflicting criteria, which can help the decision makers to reach a final decision. This abbreviation comes from the Serbian name of the method: Višekriterijumska Optimizacija I Kompromisno Resenje(multicriteria optimization and compromise solution). Here, the CS is a feasible solution that is the closest to the ideal solution, and a compromise means an agreement established by mutual concessions [21, 35, 37].

VIKOR focalizes on the specification of a set of CSs that are built by reciprocal compensations $[6,7,28,35,37]$ to efficiently help MCDA and improve the quality of DM [50]. In recent years, VIKOR method studies have acquired popularity in varied MCDA areas because of their large ability to compromise rank performance by comparing the measure of closeness to the ideal solution [21]. Because of these properties, the VIKOR method has become popular and has been used in many areas [21]. The VIKOR method usually utilizes the positive- and negative-ideal solutions to serve as points of reference, and the specification of these ideal solutions is immediately produced from the evaluative ratings of all alternatives according to various criteria $[35,37]$. Chen [12] defined the VIKOR method based on distance index for Pythagoras fuzzy clusters and applied it to various problems.

$\mathrm{PF}$ sets include more uncertainties and are generally able to accommodate higher degrees of uncertainty. To efficiently administrate the complex uncertainty in human cognitive and DM activities, it is necessary to expand the 
valid VIKOR methods that previously focalized on certain settings and fuzzy environments to the PF context. Although the VIKOR method is a very qualified approach in terms of DM processes, it has been used very little in medical DM, health services, and child development.

The aim of this study is to develop a new approach to solve MADM problems by integrating the VIKOR method with GPFSS. It is known that the importance of the VIKOR method is to determine the CS and to improve the standard of the DM process. The VIKOR method is balancing the majority's maximum group utility and the opponent's minimum individual regret. In the VIKOR method, the best and worst solutions serve as the point of reference and the distance between the best solution and evaluative ratings does not have an upper bound. The new idea of remoteness index is considerable because it ensures the upper bounds by dividing the distance between the best solution and evaluative ratings by the distance between the best solution and the worst solution. The novel ranking indexes based on the new idea of remoteness indexes are debated to ensure more efficient compromise rankings. Thus, a remoteness index-based VIKOR method for GPFSS is improved.

The negatives caused by early childhood children staying at home during quarantine times are measured with new algorithms. All areas of development are in interaction with each other, and the problem that may occur in one area has reflections on other areas. In this context, problems in the cognitive field may cause problems in other areas, as well as problems in other areas that may reflect on cognitive development. Therefore, it is necessary to know the development characteristics of each field well and to identify the problems that may arise in a timely and accurate manner. The pre-school period has a very important place in human life. In this period, the child's personality development and emotional, social, cognitive, language, and academic development are largely completed. The child completes her/his cognitive and behavioral development in a healthy way with the contribution of her family and school environment. That's why new algorithms have been applied to such an important subject.

The originality of this study can be expressed as follows: New distance measures such as Euclidean, Hamming, and Generalized for GPFFS are defined. The properties of these distance measures were examined, and an example was given. The displaced and fixed ideals are defined for PFVs. In addition, the displaced and fixed remoteness indexes are given for PFVs. Two types of weights called precise weights and PF weights are defined and discuss in their methods of generation. The precise importance weights are obtained by the expectation score function. The PF importance weights represented the importance and unimportance degrees of the criteria. The displaced and fixed ideals, the displaced and fixed remoteness indexes are identified as ranking indexes (four groups). Based on these definitions, four algorithms expressing VIKOR methods have been proposed. The problem of the cognitive and behavioral development of early childhood children in quarantine is examined with the suggested remotenessbased VIKOR method.

\section{Preliminaries}

In this section, basic definitions, theorems, propositions and properties that will be used in the whole study will be given. Throughout the study, the initial universe, parameters sets will denote $\Upsilon, \Sigma$, respectively.

\subsection{Pythagorean fuzzy environment}

The theory of IFS was developed by Atanassov [3] and is a natural generalization of the theory of FS. IFS is defined by MS and NMS degrees and, for this reason, can indicate the fuzzy character of data in more elaboration comprehensively. The significant qualification of IFS is that it appoints to each element MS and NMS degrees $(M S+N M S \leq 1)$. However, in some DM processes, the sum of the MS and NMS values obtained may be greater than 1. In this case, we can take the sum of the squares of these MS and NMS values obtained, which will be less than or equal to 1 .

As an original idea, PFSs were created by Yager [45]. PFS is a very useful tool for uncertainty. PFS offers good results especially in solutions where IFS is insufficient. The differences between PFSs and IFSs can be mentioned as follows: For IFS, $M S+N M S \leq 1,0 \leq M S+N M S \leq 1, \pi=$ $1-(M S+N M S)$ and $\pi+M S+N M S=1$.

For $\quad P F S, \quad M S+N M S \leq 1 \quad$ or $\quad M S+N M S \geq 1$, $0 \leq M S^{2}+N M S^{2} \leq 1, \quad \pi=\sqrt{1-\left(M S^{2}+N M S^{2}\right)} \quad$ and $\pi^{2}+M S^{2}+N M S^{2}=1$.

For, the initial universe set $\Upsilon$, the function $u_{A}(x): \Upsilon \rightarrow$ $[0,1]$ is called $F S$ on $\Upsilon$. The FS can be indicated by

$A=\left\{\left(a_{i}, u_{A}\left(a_{i}\right)\right): u_{A}\left(a_{i}\right) \in[0,1] ; \forall a_{i} \in \Upsilon\right\}$.

Choose the MS function $u_{B}: \Upsilon \rightarrow[0,1]$ and the NMS function $v_{B}: \Upsilon \rightarrow[0,1]$. Let's assume that the condition $0 \leq u_{B}(a)+v_{B}(a) \leq 1$ for any $a \in \Upsilon$ is satisfied. Then, the set $B=\left\{\left(a, u_{B}(a), v_{B}(a)\right): a \in \Upsilon\right\}$ is said to be an IFS $B$ on $\Upsilon$. In this case, the condition $\pi_{B}=1-u_{B}(x)-v_{B}(x)$ holds [3].

Suppose that condition $0 \leq\left[u_{C}(x)\right]^{2}+\left[v_{C}(x)\right]^{2} \leq 1$ satisfies for $u_{C}: \Upsilon \rightarrow[0,1]$ and $v_{C}: \Upsilon \rightarrow[0,1]$. Then, an PFS $C$ in $\Upsilon$ is defined by $C=\left\{\left(x, u_{C}(x), v_{C}(x)\right): x \in \Upsilon\right\}$. In 
this case, the condition $\pi_{C}=\sqrt{1-\left[u_{C}(x)\right]^{2}-\left[v_{C}(x)\right]^{2}}$ holds [45, 46, 48].

Let $C$ be a PFS over $\Sigma$. In this definition, $(F, C)$ can be represented as

$$
\begin{aligned}
(F, C)= & \left\{\left(a, u_{F}(a), v_{F}(a)\right):\right. \\
& \left.a \in \mathscr{P}, u_{F}(a) \in[0,1], v_{F}(a) \in[0,1]\right\} .
\end{aligned}
$$

The set of all PFS on $\Upsilon$ will be denoted by $\Omega(\Upsilon)$.

Let $\Upsilon, \Sigma$ be an initial universe and a parameters sets, respectively. For $D \subseteq \Sigma$, consider a set-valued mapping $F: D \rightarrow \omega(\Upsilon)$, where the power set of $\Upsilon$ is showed by $\omega(\Upsilon)$. Therefore, a pair $(F, D)$ is called a SS on $\Upsilon$.

For $E \subseteq \Sigma$, choose $F: E \rightarrow \omega(\Upsilon)$, where the set of all PFSs over $\Upsilon$ is indicated by $\omega(\Upsilon)$. Then, a pair $(F, E)$ is called a PFSS on $\omega(\Upsilon)$ [38].

Choose the Pythagorean fuzzy numbers (PFNs) $R=$ $\left(u_{R}, v_{R}\right) \quad$ [51]. $\quad$ For three PFNs $\theta=(u, v), \theta_{1}=\left(u_{1}, v_{1}\right), \theta_{2}=\left(u_{2}, v_{2}\right)$, some fundamental operations are as follows [45, 48]: For $\alpha>0, \bar{\theta}=(v, u)$, $\theta_{1} \oplus \theta_{2}=\left(\sqrt{u_{1}^{2}+u_{2}^{2}-u_{1}^{2} u_{2}^{2}}, v_{1} v_{2}\right)$,

$\theta_{1} \otimes \theta_{2}=\left(u_{1} u_{2}, \sqrt{v_{1}^{2}+v_{2}^{2}-v_{1}^{2} v_{2}^{2}}\right)$,

$\theta_{1} \wedge \theta_{2}=\left(\min \left\{u_{1}, u_{2}\right\}, \max \left\{v_{1}, v_{2}\right\}\right)$,

$\theta_{1} \vee \theta_{2}=\left(\max \left\{u_{1}, u_{2}\right\}, \min \left\{v_{1}, v_{2}\right\}\right)$,

$\alpha \cdot \theta=\left(\sqrt{1-\left(1-u^{2}\right)^{\alpha}}, v^{\alpha}\right), \quad \theta^{\alpha}=\left(u^{\alpha}, \sqrt{1-\left(1-v^{2}\right)^{\alpha}}\right)$.

The PFS defined by MS and NMS which satisfies the condition $M S^{2}+N M S^{2} \leq 1$ is initiated by Yager [45]. For example, choose $\quad p=(0.87,0.42)$ Since $(0,87)+(0.42)>1$, these values are not suitable for IFS. However, since $(0,87)^{2}+(0.42)^{2}<1$, these values can be used with PFS.

A new PFN formula consisting of the strength of commitment $r_{p}$ and the direction of commitment $d_{p}$ was also given by Yager [45]. The new PFN is denoted by $P=\left(r_{p}, d_{p}\right)$, where $r_{p} \in[0,1]$. The stronger commitment is defined by the larger the value of $r_{p}$, in this case the lower the uncertainty of the commitment. $r_{p}$ and $d_{p}$ are characterized by $u_{p}=r_{p} \cos \left(\theta_{p}\right), v_{p}=r_{p} \sin \left(\theta_{p}\right)$, i.e., these values are related to MS and NMS grades. Here $\theta_{p}$ is expressed in radian and calculate $\theta_{p}=\left(1-d_{p}\right)(\pi / 2)$. If $P=\left(r_{p}, d_{p}\right)$ is defined in terms of polar coordinates, it is denoted as $\left(r_{p}, \theta_{p}\right)$. In this case, $d_{p}$ has a formula in the form of $d_{p}=1-\left(2 . \theta_{p} / \pi\right)$. It can then be said that PFNs consists of $u_{p}, v_{p}, \pi_{p}, r_{p}, d_{p}$ and $\theta_{p}$ parameters. If $u_{p}, v_{p} \in[0,1]$, then it is clear that $u_{p}^{2} \leq u_{p}, v_{p}^{2} \leq v_{p}$.

\subsection{Generalized Pythagorean fuzzy soft sets}

Consider the $L=\left\{(a, b): a, b \in[0,1], a^{2}+b^{2}<1\right\}$ with the partial order $\leq_{L}$. The complete lattice $\left(L, \leq_{L}\right)$ is defined by $(a, b) \leq_{L}(i, j) \quad \Leftrightarrow \quad a \leq i$ and $b \geq j$, for all $(a, b),(i, j) \in L$.

The $(a, b) \in \mathscr{L}$ given in this way is called Pythagorean fuzzy value $(\mathrm{PFN})$ (or Pythagorean fuzzy number(PFN)) [16].

Definition 2.1 [25] Take the PFS $C$. Let the PFS $f: C \rightarrow L$. If the set

$$
\begin{aligned}
& (F, C, f)=\left\{\left(a, u_{C}(a), v_{C}(a), f_{C}(a)\right):\right. \\
& \left.\quad a \in \sum, f_{C}(a) \in L, u_{C}(a) \in[0,1], v_{C}(a) \in[0,1]\right\}
\end{aligned}
$$

is a PFSS, the $(F, C, f)$ is called Pythagorean fuzzy parameterized Pythagorean fuzzy soft set (generalized Pythagorean fuzzy soft set-GPFSS), where $(F, C)$ is a fundamental PFSS (FPFSS). Furthermore, $f_{C}$ is said to be the parametric PFS(PPFS) and is indicated by $u_{f}, v_{f}$.

Example 2.2 Choose the patients set $H=\{h 1, h 2, h 3\}$ and the symptoms set $B=\left\{b_{1}, b_{2}, b_{3}, b_{4}\right\} \quad$ and $C=\left\{b_{1}, b_{3}, b_{4}\right\} \subset B$. The values $f_{C}\left(b_{1}\right)=(0.77,0.35)$, $f_{C}\left(b_{3}\right)=(0.65,0.42)$ and $f_{C}\left(b_{4}\right)=(0.88,0.24)$ express the diagnostic view of a physician. The $(F, C, f)$ is shown in Table 1.

For all $R=\left(u_{R}, v_{R}\right) \in L$, the score function for PFNs ie described

$S C_{R}=u_{R}^{2}-v_{R}^{2}$

where $S C: L \rightarrow[-1,1][1,51]$.

For any two PFNs $R, T$,

$\left\{\begin{aligned} R \prec T, & \text { if } & \mathscr{S} \mathscr{C}(R) & <\mathscr{S} \mathscr{C}(T), \\ R \succ T, & \text { if } & \mathscr{S} \mathscr{C}(R) & >\mathscr{S} \mathscr{C}(T), \\ R \sim T, & \text { if } & \mathscr{S} \mathscr{C}(R) & =\mathscr{S} \mathscr{C}(T) ;\end{aligned}\right.$

Table 1 The GPFSS $(F, C, f)$

\begin{tabular}{llll}
\hline & $b_{1}$ & $b_{3}$ & $b_{4}$ \\
\hline$h_{1}$ & $(0.71,0.66)$ & $(0.58,0.48)$ & $(0.82,0.30)$ \\
$h_{2}$ & $(0.55,0.70)$ & $(0.92,0.21)$ & $(0.63,0.56)$ \\
$h_{3}$ & $(0.94,0.13)$ & $(0.76,0.37)$ & $(0.70,0.44)$ \\
$f_{C}\left(b_{i}\right)$ & $(0.77,0.35)$ & $(0.65,0.42)$ & $(0.88,0.24)$ \\
\hline
\end{tabular}


As can be seen from (2.4), the larger the score $s_{R}$, the greater the PFN $R$ [16]. It should be noted that $\mathscr{S} \mathscr{C}$ cannot differentiate some evidently distinct PFNs that have the same score. To explain this situation, we can give examples using (2.5): Take two PFNs $R, T$ as $R=(0.481,0.402)$ and $T=(0.527,0.456) . \quad$ Then, $\quad S C_{R}=0.0697 \quad$ and $S C_{T}=0.0697$. Again, for $R=(0.123,0.123)$ and $T=$ $(0.456,0.456)$ we can write $R \sim T$. Therefore, if only the scoring function is used for comparison, it is not possible to make a comparison between these numbers. To overcome this problem, we can define a new function [1], as follows:

The mapping $\mathscr{A} \mathscr{C}: \mathscr{L} \rightarrow[0,1]$ is called accuracy function, if

$\mathscr{A} \mathscr{C}_{R}=u_{R}^{2}+v_{R}^{2}$

for all $R=\left(u_{R}, v_{R}\right) \in \mathscr{L}$ [39].

Using the (2.4) and (2.6), for comparing PFVs, the following method is presented by Agarwal et al. [1]. For any two PFNs $R$, $T$, if $\mathscr{S} \mathscr{C}(R)=\mathscr{S} \mathscr{C}(T)$, then

$$
\left\{\begin{array}{cc}
R>T, \text { if } & \mathscr{A} \mathscr{C}(R)>\mathscr{A} \mathscr{C}(T), \\
R<T, \text { if } & \mathscr{A} \mathscr{C}(R)<\mathscr{A} \mathscr{C}(T) \\
R \sim T, \text { if } & \mathscr{A} \mathscr{C}(R)=\mathscr{A} \mathscr{C}(T) ;
\end{array}\right.
$$

For a binary relation $\leq_{(\mathscr{S} \mathscr{C}, \mathscr{A} \mathscr{C})} \in \mathscr{L}$ and $R, T \in \mathscr{L}$, it can be written as

$$
\begin{aligned}
R & \leq(\mathscr{S} \mathscr{C}, \mathscr{A} \mathscr{C}) T \quad \Leftrightarrow \quad\left(\mathscr{S} \mathscr{C}_{R}<\mathscr{S} \mathscr{C}_{T}\right) \vee\left(\mathscr{S} \mathscr{C}_{R}\right. \\
& \left.=\mathscr{S} \mathscr{C}_{T} \wedge \mathscr{A} \mathscr{C}_{R} \leq \mathscr{A} \mathscr{C}_{T}\right) .
\end{aligned}
$$

From [14], we can give the new definition:

Definition 2.3 [27] The mapping $\mathscr{E} \mathscr{S}: \mathscr{L} \rightarrow[0,1]$ is called expectation score function such that for all $R=\left(m_{R}, n_{R}\right) \in \mathscr{L}$

$$
\mathscr{E} \mathscr{S}_{R}=\frac{u_{R}^{2}-v_{R}^{2}+1}{2} \text {. }
$$

Proposition 2.4 [27] Let $\mathscr{E} \mathscr{S}: \mathscr{L} \rightarrow[0,1]$ and $R=\left(u_{R}, v_{R}\right) \in \mathscr{L}$. Then, we have

i. $\mathscr{E} \mathscr{S}(0,1)=0$ and $\mathscr{E} \mathscr{S}(1,0)=1$,

ii. $\mathscr{E} \mathscr{S}\left(u_{R}, v_{R}\right)$ is increasing with respect to $u_{R}$,

iii. $\mathscr{E} \mathscr{S}\left(u_{R}, v_{R}\right)$ is decreasing with respect to $v_{R}$.

Definition 2.5 [27] For two PFNs $R, T \in \mathscr{L}$ and the relation $\leq_{(u, \mathscr{E} \mathscr{S})}$ on $\mathscr{L}$, we have

$$
\begin{aligned}
R & \leq{ }_{(u, \mathscr{E} \mathscr{S})} T \quad \Leftrightarrow \quad\left(u_{R}<u_{T}\right) \vee\left(u_{R}\right. \\
& \left.=u_{T} \wedge \mathscr{E} \mathscr{S}_{R} \leq \mathscr{E} \mathscr{S}_{T}\right) .
\end{aligned}
$$

Theorem 2.6 [27] The relation $\leq_{(u, \mathscr{G} \mathscr{S})}$ is a partial order on $\mathscr{L}$.

By replacing the approval rates in Definition 2.5, the other relation $\leq_{(\mathscr{E} \mathscr{G}, u)}$ is written as follows:

Definition 2.7 [27] For two PFNs $R, T \in \mathscr{L}$ and the relation $\leq_{(\mathscr{E} \mathscr{G}, u)}$ on $\mathscr{L}$,

$$
R \leq_{(\mathscr{E} \mathscr{S}, u)} T \quad \Leftrightarrow \quad\left(\mathscr{E} \mathscr{S}_{R}<\mathscr{E} \mathscr{S}_{T}\right) \vee\left(\mathscr{E} \mathscr{S}_{R}=\mathscr{E} \mathscr{S}_{T} \wedge u_{R} \leq v_{T}\right)
$$

The relationship with each other of $\leq_{(\mathscr{P F}, \mathscr{A} \mathscr{C})}$ and $\leq{ }_{(\mathscr{E} \mathscr{S}, u)}$ can be given as follows:

Proposition 2.8 [27] Let $R$ and $T$ be PFNs in $\mathscr{L}$. Then, $R \leq{ }_{(\mathscr{S} \mathscr{C}, \mathscr{A} \mathscr{C})} T \Leftrightarrow R \leq_{(\mathscr{E} \mathscr{C}, u)} T$.

If we consider the debates of $[14,44]$, then the following proposition is given:

Proposition 2.9 [27] Take two PFVs $R=\left(u_{R}, v_{R}\right)$ and $T=\left(u_{T}, v_{T}\right)$ in $\mathscr{L}$. Then, the following conditions are equivalent:

$$
\begin{aligned}
\text { i. } & \mathscr{S} \mathscr{C}_{R}=\mathscr{S} \mathscr{C}_{T} \wedge \mathscr{A}_{R} \leq \mathscr{A} \mathscr{C}_{T}, \\
\text { ii. } & \mathscr{E} \mathscr{S}_{R}=\mathscr{E} \mathscr{S}_{T} \wedge u_{R} \leq u_{T}, \\
\text { iii. } & \mathscr{E} \mathscr{S}_{R}=\mathscr{E} \mathscr{S}_{T} \wedge v_{R} \leq v_{T}, \\
\text { iv. } & \mathscr{S} \mathscr{C}_{R}=\mathscr{S} \mathscr{C}_{T} \wedge v_{R} \leq u_{T}, \\
\text { v. } & \mathscr{S} \mathscr{C}_{R}=\mathscr{S} \mathscr{C}_{T} \wedge v_{R} \leq v_{T} .
\end{aligned}
$$

Definition 2.10 [51] Let $R=\left(u_{R}, v_{R}\right), T=\left(u_{T}, v_{T}\right) \in \mathscr{L}$ be two PFVs. Then, for $\alpha>0$, we have the following operations:

$$
\begin{aligned}
& R \oplus T=\left(\sqrt{u_{R}^{2}+u_{T}^{2}-u_{M}^{2} u_{T}^{2}}, v_{R} v_{T}\right), \\
& R \otimes T=\left(u_{R} u_{T}, \sqrt{v_{R}^{2}+v_{T}^{2}-v_{R}^{2} v_{T}^{2}}\right), \\
& \alpha R=\left(\sqrt{1-\left(1-u_{R}^{2}\right)^{\alpha}},\left(v_{R}\right)^{\alpha}\right), \\
& R^{\alpha}=\left(\left(u_{R}\right)^{\alpha}, \sqrt{1-\left(1-v_{R}^{2}\right)^{\alpha}},\right) .
\end{aligned}
$$

Theorem 2.11 [51] For $R=\left(u_{R}, v_{R}\right), T=\left(u_{T}, v_{T}\right) \in \mathscr{L}$ and $\alpha, \alpha_{1}, \alpha_{2}>0$,

$$
\begin{aligned}
\text { i. } & R \oplus T=T \oplus R, \\
\text { ii. } & R \otimes T=T \otimes R, \\
\text { iii. } & \alpha(R+T)=\alpha R \oplus \alpha T, \\
\text { iv. } & \alpha_{1} R \oplus \alpha_{2} R=\left(\alpha_{1}+\alpha_{2}\right) R, \\
\text { v. } & (R \otimes T)^{\alpha}=R^{\alpha} \otimes T^{\alpha}, \\
\text { vi. } & R^{\alpha_{1}} \oplus R^{\alpha_{2}}=R^{\left(\alpha_{1}+\alpha_{2}\right)} .
\end{aligned}
$$


Proposition 2.12 [27] For $\quad R=\left(u_{R}, v_{R}\right)$, build useful concepts of remoteness indices, consisting of

$T=\left(u_{T}, v_{T}\right), V=\left(u_{V}, v_{V}\right) \in \mathscr{L}$, then we have,

$R \leq_{(u, \mathscr{E} \mathscr{S})} V \Rightarrow T \oplus R \leq_{(u, \mathscr{E} \mathscr{S})} T \oplus V$.

Corollary 2.13 [27] Let For $R=\left(u_{R}, v_{R}\right), T=\left(u_{T}, v_{T}\right) \in$ $\mathscr{L}$ and $\alpha, \alpha_{1}, \alpha_{2} \in \mathbb{R}^{+}$. Then,

i. If $R \leq_{(u, \mathscr{E} \mathscr{S})} T$, then $\quad \alpha R \leq_{(u, \mathscr{E} \mathscr{S})} \alpha T$,

ii. If $\alpha_{1} \leq \alpha_{2}$, then $\alpha_{1} R \leq_{(u, \mathscr{E} \mathscr{S})} \alpha_{2} R$.

\section{Distance measures for GPFSSs}

The Hamming, Euclidean and generalized distance measures for GPFSSs are given in this section. Some properties of distance measures are discussed. The new distance measures offered in this section will be then applied to displaced positive- and negative-ideal remoteness indices as well as fixed positive- and negative-ideal remoteness indices.

Let $\Upsilon=\left\{x_{1}, x_{2}, \cdots, x_{m}\right\}, \sum=\left\{e_{1}, e_{2}, \cdots, e_{n}\right\}$ be universe set and parameter set, respectively. Choose the two GPFSSs $\Omega_{1}, \Omega_{2}$. For convenience, take $\triangle X\left(x_{i}\right)=X\left(\Omega_{1}\right)_{e j}\left(x_{i}\right)-X\left(\Omega_{2}\right)_{e j}\left(x_{i}\right)$.

Definition 3.1 For two GPFSSs $\Omega_{1}=(F, A, f)$, $\Omega_{2}=(G, B, g)$, the normalized Hamming distance, normalized Euclidean distance and generalized distance measures between $\Omega_{1}$ and $\Omega_{2}$ are defined, respectively, as follows:

$$
U_{H}\left(\Omega_{1}, \Omega_{2}\right)=\frac{1}{5 m n}\left\{\begin{array}{c}
\sum_{i=1}^{m} \sum_{j=1}^{n}\left[\left|\Delta u\left(x_{i}\right)\right|+\left|\Delta v\left(x_{i}\right)\right|+\left|\Delta \pi\left(x_{i}\right)\right|+\left|\Delta r\left(x_{i}\right)\right|+\left|\Delta d\left(x_{i}\right)\right|\right. \\
+\left|u(f)_{e_{j}}\left(x_{i}\right)-u(g)_{e_{j}}\left(x_{i}\right)\right|+\left|v(f)_{e_{j}}\left(x_{i}\right)-v(g)_{e_{j}}\left(x_{i}\right)\right|+\left|\pi(f)_{e_{j}}\left(x_{i}\right)-\pi(g)_{e_{j}}\left(x_{i}\right)\right| \\
\left.+\left|r(f)_{e_{j}}\left(x_{i}\right)-r(g)_{e_{j}}\left(x_{i}\right)\right|+\left|d(f)_{e_{j}}\left(x_{i}\right)-d(g)_{e_{j}}\left(x_{i}\right)\right|\right]
\end{array}\right\}
$$

$$
U_{E}\left(\Omega_{1}, \Omega_{2}\right)=\left\{\begin{array}{c}
\frac{1}{5 m n} \sum_{i=1}^{m} \sum_{j=1}^{n}\left[\left(\Delta u\left(x_{i}\right)\right)^{2}+\left(\Delta v\left(x_{i}\right)\right)^{2}+\left(\Delta \pi\left(x_{i}\right)\right)^{2}+\left(\Delta r\left(x_{i}\right)\right)^{2}\right. \\
+\left(\Delta d\left(x_{i}\right)\right)^{2}+\left(u(f)_{e_{j}}\left(x_{i}\right)-u(g)_{e_{j}}\left(x_{i}\right)\right)^{2}+\left(v(f)_{e_{j}}\left(x_{i}\right)-v(g)_{e_{j}}\left(x_{i}\right)\right)^{2} \\
+\left(\pi(f)_{e_{j}}\left(x_{i}\right)-\pi(g)_{e_{j}}\left(x_{i}\right)\right)^{2}+\left(r(f)_{e_{j}}\left(x_{i}\right)-r(g)_{e_{j}}\left(x_{i}\right)\right)^{2} \\
\left.+\left(d(f)_{e_{j}}\left(x_{i}\right)-d(g)_{e_{j}}\left(x_{i}\right)\right)^{2}\right]
\end{array}\right\}^{1 / 2}
$$

$$
U_{G}\left(\Omega_{1}, \Omega_{2}\right)=\left\{\begin{array}{c}
\frac{1}{5 m n} \sum_{i=1}^{m} \sum_{j=1}^{n}\left(\Delta u\left(x_{i}\right)\right)^{\lambda}+\left(\Delta v\left(x_{i}\right)\right)^{\lambda}+\left(\Delta \pi\left(x_{i}\right)\right)^{\lambda} \\
+\left(\Delta d\left(x_{i}\right)\right)^{\lambda}+\left(\Delta r\left(x_{i}\right)\right)^{\lambda}+\left(u(f)_{e_{j}}\left(x_{i}\right)-u(g)_{e_{j}}\left(x_{i}\right)\right)^{\lambda} \\
+\left(v(f)_{e_{j}}\left(x_{i}\right)-v(g)_{e_{j}}\left(x_{i}\right)\right)^{\lambda}+\left(\pi(f)_{e_{j}}\left(x_{i}\right)-\pi(g)_{e_{j}}\left(x_{i}\right)\right)^{\lambda} \\
+\left(r(f)_{e_{j}}\left(x_{i}\right)-r(g)_{e_{j}}\left(x_{i}\right)\right)^{\lambda}+\left(d(f)_{e_{j}}\left(x_{i}\right)-d(g)_{e_{j}}\left(x_{i}\right)\right)^{\lambda}
\end{array}\right\}
$$


where $\lambda \geq 1$.

Definition 3.2 The weighted normalized Hamming distance, weighted normalized Euclidean distance and weighted generalized distance measures between $\Omega_{1}$ and $\Omega_{2}$ are defined, respectively, as follows: order $\leq_{L}$. A generalized distance measure of $U_{G}$ satisfies the following conditions:

$\left(U_{1}\right) \quad U_{G}\left(\Omega_{1}, \Omega_{1}\right)=0$ (reflexivity)

$\left(U_{2}\right) \quad U_{G}\left(\Omega_{1}, \Omega_{2}\right)=0$ if $\Omega_{1}=\Omega_{2}$

$\left(U_{3}\right) \quad U_{G}\left(\Omega_{1}, \Omega_{2}\right)=U_{G}\left(\Omega_{2}, \Omega_{1}\right)$ (symmetry)

$\left(U_{4}\right) \quad 0 \leq U_{G}\left(\Omega_{1}, \Omega_{1}\right) \leq 1$ (boundedness)

$U_{W H}\left(\Omega_{1}, \Omega_{2}\right)=\frac{1}{5 m n}\left\{\begin{array}{c}\sum_{i=1}^{m} \sum_{j=1}^{n} \omega_{j}\left|\Delta u\left(x_{i}\right)\right|+\left|\Delta v\left(x_{i}\right)\right|+\left|\Delta \pi\left(x_{i}\right)\right|+\left|\Delta r\left(x_{i}\right)\right| \\ +\left|\Delta d\left(x_{i}\right)\right|+\left|u(f)_{e_{j}}\left(x_{i}\right)-u(g)_{e_{j}}\left(x_{i}\right)\right| \\ +\left|v(f)_{e_{j}}\left(x_{i}\right)-v(g)_{e_{j}}\left(x_{i}\right)\right|+\left|\pi(f)_{e_{j}}\left(x_{i}\right)-\pi(g)_{e_{j}}\left(x_{i}\right)\right| \\ +\left|r(f)_{e_{j}}\left(x_{i}\right)-r(g)_{e_{j}}\left(x_{i}\right)\right|+\left|d(f)_{e_{j}}\left(x_{i}\right)-d(g)_{e_{j}}\left(x_{i}\right)\right|\end{array}\right\}$

$U_{W E}\left(\Omega_{1}, \Omega_{2}\right)=\left\{\begin{array}{c}\frac{1}{5 m n} \sum_{i=1}^{m} \sum_{j=1}^{n} \omega_{j}\left(\Delta u\left(x_{i}\right)\right)^{2}+\left(\Delta v\left(x_{i}\right)\right)^{2}+\left(\Delta \pi\left(x_{i}\right)\right)^{2}+\left(\Delta r\left(x_{i}\right)\right)^{2} \\ +\left(\Delta d\left(x_{i}\right)\right)^{2}+\left(u(f)_{e_{j}}\left(x_{i}\right)-u(g)_{e_{j}}\left(x_{i}\right)\right)^{2} \\ +\left(v(f)_{e_{j}}\left(x_{i}\right)-v(g)_{e_{j}}\left(x_{i}\right)\right)^{2}+\left(\pi(f)_{e_{j}}\left(x_{i}\right)-\pi(g)_{e_{j}}\left(x_{i}\right)\right)^{2} \\ +\left(r(f)_{e_{j}}\left(x_{i}\right)-r(g)_{e_{j}}\left(x_{i}\right)\right)^{2}+\left(d(f)_{e_{j}}\left(x_{i}\right)-d(g)_{e_{j}}\left(x_{i}\right)\right)^{2}\end{array}\right\}$

$U_{W G}\left(\Omega_{1}, \Omega_{2}\right)=\left\{\begin{array}{c}\frac{1}{5 m n} \sum_{i=1}^{m} \sum_{j=1}^{n} \omega_{j}\left(\Delta u\left(x_{i}\right)\right)^{\lambda}+\left(\Delta v\left(x_{i}\right)\right)^{\lambda}+\left(\Delta \pi\left(x_{i}\right)\right)^{\lambda}+\left(\Delta r\left(x_{i}\right)\right)^{\lambda} \\ +\left(\Delta d\left(x_{i}\right)\right)^{\lambda}+\left(u(f)_{e_{j}}\left(x_{i}\right)-u(g)_{e_{j}}\left(x_{i}\right)\right)^{\lambda} \\ +\left(v(f)_{e_{j}}\left(x_{i}\right)-v(g)_{e_{j}}\left(x_{i}\right)\right)^{\lambda}+\left(\pi(f)_{e_{j}}\left(x_{i}\right)-\pi(g)_{e_{j}}\left(x_{i}\right)\right)^{\lambda} \\ +\left(r(f)_{e_{j}}\left(x_{i}\right)-r(g)_{e_{j}}\left(x_{i}\right)\right)^{\lambda}+\left(d(f)_{e_{j}}\left(x_{i}\right)-d(g)_{e_{j}}\left(x_{i}\right)\right)^{\lambda}\end{array}\right\}$

where $\lambda \geq 1,0 \leq \omega_{i} \leq 1(i=1,2, \cdots, n)$ and $\sum_{i=1}^{n} \omega_{i}=1$.

Theorem 3.3 Let $(\mathscr{L}, \leq \mathscr{L})$ be a partially ordered set. For the GPFSSs $\Omega_{1}, \Omega_{2}, \Omega_{3}$ belonging to $L$ with the partial
$\left(U_{5}\right)$

$$
U_{G}\left(\Omega_{1}, \Omega_{2}\right) \leq U_{G}\left(\Omega_{1}, \Omega_{3}\right)
$$

and $U_{G}\left(\Omega_{2}, \Omega_{3}\right) \leq U_{G}\left(\Omega_{1}, \Omega_{3}\right)$ if $p_{1} \leq_{L} p_{2} \leq_{L} p_{3}$.

The proof of this theorem is obvious. 
Note 1: If $\lambda=1$ and $\lambda=2, U_{H}=U_{G}$ and $U_{E}=U_{G}$, respectively. Then $U_{H}$ satisfies the conditions of Theorem 3.3 with separability and triangle inequality. Therefore, the Hamming distance measure $U_{H}$ is a metric. Further, $U_{E}$ satisfies the conditions of Theorem 3.3 with separability. Fulfilling these conditions indicates that the $U_{E}$ is a semi-metric. The conditions separability and triangle inequality conditions, respectively, are as follows:

$\left(A_{1}\right) \quad U_{G}\left(\Omega_{1}, \Omega_{2}\right)=0 \Leftrightarrow \Omega_{1}=\Omega_{2}$, for $\lambda=1$ and $\lambda=2$.

$\left(A_{1}\right) \quad U_{H}\left(\Omega_{1}, \Omega_{3}\right) \leq U_{H}\left(\Omega_{1}, \Omega_{2}\right)+U_{H}\left(\Omega_{2}, \Omega_{3}\right)$

Theorem 3.4 For the GPFSSs $\Omega_{1}, \Omega_{2}, \quad U\left(\Omega_{1}, \Omega_{2}\right)$ $=U\left(\Omega_{1}^{t}, \Omega_{2}^{t}\right)$.

Proof We will only prove for normalized Hamming distance. It can be proved similarly for normalized Euclidean distance and generalized distance measures. The distance between two GPFSSs $\Omega_{1}, \Omega_{2}$ can be written as follows: the MS degrees of $\Omega_{1}^{t}$ relate to the NMS degrees of $\Omega_{2}^{t}$. Therefore, the distance between $\Omega_{1}, \Omega_{2}$ is the same as the distance between $\Omega_{1}^{t}, \Omega_{2}^{t}$.

Example 3.5 Let $\Upsilon=\left\{x_{1}, x_{2}\right\}, M=N=\left\{e_{1}, e_{2}, e_{3}\right\}$ be universe set and parameter sets, respectively. Choose two GPFFSs $\Omega_{1}=(F, M, f), \Omega_{2}=(G, N, g)$ as follows:

$$
U_{H}\left(\Omega_{1}, \Omega_{2}\right)=\frac{1}{5 m n}\left\{\begin{array}{c}
\sum_{i=1}^{m} \sum_{j=1}^{n}\left[\left|u\left(\Omega_{1}\right)_{e j}\left(x_{i}\right)-u\left(\Omega_{2}\right)_{e j}\left(x_{i}\right)\right|+\left|v\left(\Omega_{1}\right)_{e j}\left(x_{i}\right)-v\left(\Omega_{2}\right)_{e j}\left(x_{i}\right)\right|\right. \\
+\left|\pi\left(\Omega_{1}\right)_{e j}\left(x_{i}\right)-\pi\left(\Omega_{2}\right)_{e j}\left(x_{i}\right)\right|+\left|r\left(\Omega_{1}\right)_{e j}\left(x_{i}\right)-r\left(\Omega_{2}\right)_{e j}\left(x_{i}\right)\right| \\
+\left|d\left(\Omega_{1}\right)_{e j}\left(x_{i}\right)-d\left(\Omega_{2}\right)_{e j}\left(x_{i}\right)\right|+\left|u(f)_{e_{j}}\left(x_{i}\right)-u(g)_{e_{j}}\left(x_{i}\right)\right| \\
+\left|v(f)_{e_{j}}\left(x_{i}\right)-v(g)_{e_{j}}\left(x_{i}\right)\right|+\left|\pi(f)_{e_{j}}\left(x_{i}\right)-\pi(g)_{e_{j}}\left(x_{i}\right)\right| \\
\left.+\left|r(f)_{e_{j}}\left(x_{i}\right)-r(g)_{e_{j}}\left(x_{i}\right)\right|+\left|d(f)_{e_{j}}\left(x_{i}\right)-d(g)_{e_{j}}\left(x_{i}\right)\right|\right]
\end{array}\right\}
$$

From the complement of GPFSS,

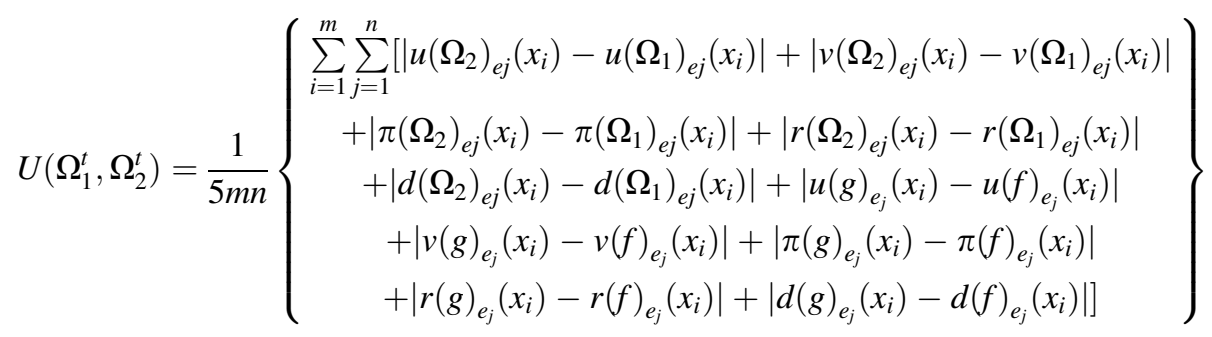




$$
\begin{aligned}
F\left(e_{1}\right) & =\left\{<x_{1}, 0.72,0.55>,<x_{2}, 0.68,0.61>\right\} \\
F\left(e_{2}\right) & =\left\{<x_{1}, 0.84,0.39>,<x_{2}, 0.75,0.65>\right\} \\
F\left(e_{3}\right) & =\left\{<x_{1}, 0.91,0.27>,<x_{2}, 0.83,0.45>\right\} \\
f & =\left\{<e_{1}, 0.48,0.83>,<e_{2}, 0.91,0.38>,\right. \\
& \left.<e_{3}, 0.69,0.54>\right\} \\
G\left(e_{1}\right) & =\left\{<x_{1}, 0.66,0.55>,<x_{2}, 0.76,0.34>\right\} \\
G\left(e_{2}\right) & =\left\{<x_{1}, 0.77,0.52>,<x_{2}, 0.83,0.25>\right\} \\
G\left(e_{3}\right) & =\left\{<x_{1}, 0.80,0.37>,<x_{2}, 0.74,0.56>\right\} \\
g & =\left\{<e_{1}, 0.46,0.63>,<e_{2}, 0.82,0.56>,\right. \\
& \left.<e_{3}, 0.67,0.47>\right\}
\end{aligned}
$$

Hamming distance measure is calculated as follows:
CS can be admitted because it ensures a maximum group utility of the majority and a minimum of the individual regret of the opponent. The CSs can be the basis for negotiations, involving the decision maker's preference on criteria weights. In the VIKOR method, the performance ratings of the alternatives according to a set of criteria are quantified as crisp values [11].

In this section, we define the displaced positive ideal PFV (DPI) and the displaced negative ideal PFV (DNI) which help to define the concept of displaced remoteness index. The fixed remoteness index is defined based on the fixed positive ideal PFS (FPI) and the fixed negative ideal PFV (FNI). The displaced and fixed group utility, individual regret and compromise indexes are the precise

$$
\begin{aligned}
& U_{H}\left(\Omega_{1}, \Omega_{2}\right)=\frac{1}{5.3 .2} \\
& \left.\qquad \begin{array}{r}
|0.72-.66|+|0.68-0.76|+|0.84-0.77|+|0.75-0.83|+|0.91-0.80|+|0.83-0.74| \\
+|0.4232-0.512|+|0.6772-0.56|+|0.38-0.37|+|0.1225-0.5|+|0.315-0.47|+|0.33-0.38| \\
+|0.906-0.86|+|0.736-0.56|+|0.93-0.93|+|0.992-0.87|+|0.95-0.88|+|0.994-0.93| \\
+ \\
+|0.585-0.56|+|0.5331-0.73|+|0.7216-0.62||0.544-0.8|+|0.8-0.724|+|0.32-0.586| \\
+|0.48-0.46|+|0.91-0.82|+|0.69-0.67|+|0.83-0.63|+|0.38-0.56|+|0.54-0.47|
\end{array}\right] .
\end{aligned}
$$

and $U_{H}\left(\Omega_{1}, \Omega_{2}\right)=0.11$. Similarly, Euclidean distance measure $U_{E}\left(\Omega_{1}, \Omega_{2}\right)=0.14$ and for $\lambda=3$, generalized distance measure $U_{G}\left(\Omega_{1}, \Omega_{2}\right)=0.1646$.

\section{The remoteness index-based VIKOR method for GPFSSs}

The purpose of this section is to develop new remoteness index-based GPFSS-VIKOR methods that utilize the concepts of displaced and fixed remoteness indices based on new distance measures to address MCDA problems within the GPFSS environment. The definition of positive and negative ideal points for a solution is the basic idea of the VIKOR method. It focalizes on ranking and selecting from a finite set of possible alternatives in the presence of contradictory and non-measurable criteria. It interprets a multicriteria ranking index based on the nearness to the ideal solution. By evaluating the alternatives according to each criterion, a compromise ranking can be obtained when the relative closeness measure is compared with the ideal alternative. Thus, the derived compromise solution is a feasible solution, which is the closest to the positive ideal solution and farthest from the negative ideal solution, and a compromise means an agreement established by mutual concessions made between the alternatives. The acquired importance and Pythagorean fuzzy importance weights. The extra parameter parametric Pythagorean fuzzy set in GPFSS, which given by the head or director of decisionmaking committee, is used to define the precise importance and Pythagorean fuzzy importance weights. Each algorithm shows the complete procedure of the remotenessbased VIKOR method based on the displaced and fixed terminologies.

Let's give the general framework of the MCDA problem about $\mathrm{PF}$ information:

Choose the set of candidate alternatives $D=\left\{d_{1}, d_{2}, \cdots, d_{m}\right\}, m(m \geq 2)$ and the set of criteria $K=$ $\left\{k_{1}, k_{2}, \cdots, k_{n}\right\} n(n \geq 2)$ as a discrete set of $m$ and a finite set of $n$, respectively. Usually, the criteria set $K$ is divided into two disjoint sets as $K_{i}$ and $K_{i i}$. $K_{i}$ and $K_{i i}$ refer to a collection of benefit criteria and cost criteria, respectively, which means a larger value better performance and a larger value worse performance, respectively. The assessment rating of alternative $d_{i} \in D$ with respect to criterion $k_{j} \in K$ is stated as a PF value $p_{i j}=\left(u_{i j}, v_{i j}\right) \cdot u_{i j}$ and $v_{i j}$ indicate the degrees that alternative $d_{i}$ satisfies and dissatisfies, respectively, criterion $k_{j}$. The hesitation degree $\pi_{i j}$ that corresponds to each $p_{i j}$ is calculated as $\pi_{i j}=\sqrt{1-\left(u_{i j}\right)^{2}-\left(v_{i j}\right)^{2}}$. Describing this situation, the $\mathrm{PF}$ matrix is as follows: 
$p_{i j}=\left[\begin{array}{cccc}\left(u_{11}, v_{11}\right) & \left(u_{12}, v_{12}\right) & \cdots & \left(u_{1 n}, v_{1 n}\right) \\ \left(u_{21}, v_{21}\right) & \left(u_{22}, v_{22}\right) & \cdots & \left(u_{2 n}, v_{2 n}\right) \\ \vdots & \vdots & \vdots & \vdots \\ \left(u_{m 1}, v_{m 1}\right) & \left(u_{m 2}, v_{m 2}\right) & \cdots & \left(u_{m n}, v_{m n}\right)\end{array}\right]$

In the PF environment, the importance weight of criterion $k_{j} \in K$ is stated as a PF value $w_{j}=\left(\bar{w}_{j}, \underline{w}_{j}\right)$, where $\bar{w}_{j}$ and $\underline{w}_{j}$ denote the degrees of importance and unimportance, respectively, of $k_{j}$. Then the hesitation degree $\tau_{j}=\sqrt{1-\bar{w}_{j}^{2}-\underline{w}_{j}^{2}}$.

\subsection{The displaced and fixed ideal IF values}

The appropriate reference points need to be designated for the PF decision process. These points will help define displaced and fixed remoteness indices. In other words, locating the PIPF and NIPF values from two different perspectives of displaced and fixed ideals is the main problem. Here, PIPF and NIPF values will be used as points of reference because these points can effectively form anchored judgments in subjective decision-making processes. To be more precise, for the anchored judgments, the displaced PIPF and NIPF values, which consist of all the best and worst criterion values, respectively, will be defined.

Firstly in light of anchored judgments with displaced ideals, the characterization of the PIPF and NIPF values with respect to each criterion can be identified according to all of the assessment ratings in the PF decision matrix $p$. This means that the PIPF and NIPF values can be often displaced because they are sensitive to changes in the available set of candidate alternatives.

Definition 4.1 Take a PF decision matrix $p=\left[p_{i j}\right]_{(m \times n)}$. For the each criterion $k_{j} \in K\left(K=K_{i} \cup K_{i i}\right)$, the displaced positive-ideal and negative-ideal PF values are defined as follows:

$$
\begin{aligned}
p(+)_{j} & =\left(u(+)_{j}, v(+)_{j}\right) \\
& = \begin{cases}\left(\max _{i=1}^{m} u_{i j}, \min _{i=1}^{m} v_{i j}\right), & \text { if } k_{j} \in K_{i} \\
\left(\min _{i=1}^{m} u_{i j}, \max _{i=1}^{m} v_{i j}\right), & \text { if } k_{j} \in K_{i i}\end{cases} \\
p(-)_{j} & =\left(u(-)_{j}, v(-)_{j}\right) \\
& = \begin{cases}\left(\min _{i=1}^{m} u_{i j}, \max _{i=1}^{m} v_{i j}\right), & \text { if } k_{j} \in K_{i} \\
\left(\max _{i=1}^{m} u_{i j}, \min _{i=1}^{m} v_{i j}\right), & \text { if } k_{j} \in K_{i i}\end{cases}
\end{aligned}
$$

and also

$$
\pi(+)_{j}=\sqrt{1-\left(u(+)_{j}\right)^{2}-\left(v(+)_{j}\right)^{2}}
$$

$$
\pi(-)_{j}=\sqrt{1-\left(u(-)_{j}\right)^{2}-\left(v(-)_{j}\right)^{2}}
$$

Consider anchored judgments with fixed ideals. It is convenient to designate $(1,0)$ and $(0,1)$ as PIPF and NIPF values, respectively, with respect to benefit criteria, because $(1,0)$ and $(0,1)$ are the top and bottom elements, respectively, of the lattice $(\mathscr{L}, \leq \mathscr{L})$. Conversely, $(0,1)$ and $(1,0)$ can be considered the PIPF and NIPF values, respectively, with respect to cost criteria for simplicity. These ideal PF values are called fixed ideal PF values.

Definition 4.2 Take a PF decision matrix $p=\left[p_{i j}\right]_{(m \times n)}$. For the each criterion $k_{j} \in K\left(K=K_{i} \cup K_{i i}\right)$, the displaced PIPF and NIPF values are defined as follows:

$$
\begin{aligned}
& p(\widehat{+})_{j}=\left(u(\widehat{+})_{j}, v(\widehat{+})_{j}\right)= \begin{cases}(1,0) & , \quad \text { if } k_{j} \in K_{i} \\
(0,1) & , \text { if } k_{j} \in K_{i i}\end{cases} \\
& p(\widehat{=})_{j}=\left(u(\widehat{=})_{j}, v(\widehat{=})_{j}\right)= \begin{cases}(0,1), & \text { if } k_{j} \in K_{i} \\
(1,0) & , \quad \text { if } k_{j} \in K_{i i}\end{cases}
\end{aligned}
$$

and also $\pi(\widehat{+})_{j}=\pi(\widehat{-})_{j}=0$.

\subsection{Remoteness index (RI)}

In this subsection, it will be constructed the displaced and fixed RIs.

Usually, the larger and the smaller the assessment rating $p_{i j}$ is, the greater the preference is for the criteria $k_{j} \in K_{i}$ and $k_{j} \in K_{i i}$, respectively. Therefore the displaced PIPF value $p(+)_{j}$ and the fixed PIPF value $p(\hat{+})_{j}$ can be deemed the most favorable values for $k_{j}$ on the basis of anchored judgments with displaced and fixed ideals, respectively. In this way, the characterization of the proposed displaced RI can be reasonably designated via the generalized distance measure $U_{G}\left(p_{i j}, p(+)_{j}\right)$. The smaller $U_{G}\left(p_{i j}, p(+)_{j}\right)$ value is, the closer to $p(+)_{j}$ it is, and the greater the preference is for each assessment rating $p_{i j}$. In a similar manner, the characterization of the proposed fixed RI can be defined using the generalized distance measure $U_{G}\left(p_{i j}, p(\widehat{+})_{j}\right)$. Moreover, the smaller $U_{G}\left(p_{i j}, p(\widehat{+})_{j}\right)$ value is, the closer $p(\widehat{+})_{j}$ it is, and the greater the preference is for each assessment rating $p_{i j}$.

However, it is worthwhile to mention that the $U_{G}\left(p_{i j}, p(+)_{j}\right)$ values for all $k_{j} \in K$ do not have a consistent upper bound in most situations. The main reason is that the displaced PIPF values are most favorable PF values with all of the currently considered assessment rating with respect to each criterion; thus, they are usually different among criteria. As a result, the maximal possible value of 
the generalized PF distances, i.e., the $U_{G}\left(p(-)_{j}, p(+)_{j}\right)$ values, can be regarded as the upper bounds of the $U_{G}\left(p_{i j}, p(+)_{j}\right)$ values for all $k_{j} \in K$.

Definition 4.3 Let $p_{i j}, p(+)_{j}, p(-)_{j}$ denote an assessment rating, the displaced PIPF value, and the displaced NIPF value, respectively, in the PF decision matrix $p=\left[p_{i j}\right]_{(m \times n)}$. Moreover, without loss of generality, assume that $p(-)_{j} \neq$ $p(+)_{j}$ for all $k_{j} \in K$. The displaced RI $R I\left(p_{i j}\right)$ of $p_{i j}$ based on the generalized distance measure $U_{G}$ is defined as follows:

$R I\left(p_{i j}\right)=\frac{U_{G}\left(p_{i j}, p(+)_{j}\right)}{U_{G}\left(p(-)_{j}, p(+)_{j}\right)}$

Theorem 4.4 Choose the three assessment ratings $p_{i j}, p_{i_{1} j}, p_{i_{2} j}$. The displaced RI satisfies the following properties:

i. $\quad R I\left(p_{i j}\right)=0 \Leftrightarrow p_{i j}=p(+)_{j} ;$

ii. $R I\left(p_{i j}\right)=1 \Leftrightarrow p_{i j}=p(-)_{j}$;

iii. $0 \leq R I\left(p_{i j}\right) \leq 1$;

iv. For each $k_{j} \in K_{i}, R I\left(p_{i_{2} j}\right) \leq R I\left(p_{i_{1} j}\right)$, if $p_{i_{1} j} \leq \mathscr{L} p_{i_{2} j}$;

v. For each $k_{j} \in K_{i i}, R I\left(p_{i_{1} j}\right) \leq R I\left(p_{i_{2} j}\right)$, if $p_{i_{1} j} \leq \mathscr{L} p_{i_{2} j}$.

\section{Proof}

(i) $\Rightarrow$ : If $R I\left(p_{i j}\right)=0$, then it implies that $U_{G}\left(p_{i j}, p(+)_{j}\right)=0$. According to the separability condition of Theorem 3.3, it is obtained that $p_{i j}=p(+)_{j}$.

$\Leftarrow$ If $p_{i j}=p(+)_{j}$, it is obvious that $R I\left(p_{i j}\right)=0$, because of the reflexivity condition $\left(U_{1}\right)$ of Theorem 3.3.

(ii) $\Rightarrow$ : If $R I\left(p_{i j}\right)=1$, then it implies that $U_{G}\left(p_{i j}, p(+)_{j}\right)=U_{G}\left(p(-)_{j}, p(+)_{j}\right)$. It follows that $p_{i j}=p(-)_{j}$.

$\Leftarrow$ If $p_{i j}=p(-)_{j}$, it is trivial to obtain that $R I\left(p_{i j}\right)=1$ by employing (4.8).

(iii) Applying (4.2) and (4.3), it is obvious that $u(-)_{j} \leq u_{i j} \leq u(+)_{j} \quad$ and $\quad v(-)_{j} \geq v_{i j} \geq v(+)_{j}$ for each $k_{j} \in K_{i}$. Moreover $u(+)_{j} \leq u_{i j} \leq u(-)_{j}$ and $v(+)_{j} \geq v_{i j} \geq v(-)_{j}$ for each $k_{j} \in K_{i i}$. Thus, one can easily obtain $p(-)_{j} \leq \mathscr{L} p_{i j} \leq \mathscr{L} p(+)_{j} \quad$ and $p(+)_{j} \leq \mathscr{L} p_{i j} \leq \mathscr{L} p(-)_{j}$ for $k_{j} \in K_{i}$ and $k_{j} \in K_{i i}$, respectively. According to $\left(U_{5}\right)$ of Theorem 3.3, it is known that $U_{G}\left(p_{i j}, p(+)_{j}\right) \leq\left(U_{G}\left(p(-)_{j}, p(+)_{j}\right)\right.$ and $U_{G}\left(p(+)_{j}, p_{i j}\right) \leq\left(U_{G}\left(p(+)_{j}, p(-)_{j}\right)\right.$ for $k_{i} \in$ $K_{i}$ and $k_{i} \in K_{i i}$, respectively. Next, combining the condition $U_{G}\left(p_{i j}, p(+)_{j}\right) \leq\left(U_{G}\left(p(-)_{j}, p(+)_{j}\right)\right.$ and the boundedness property of Theorem 3.3, one can obtain $0 \leq R I\left(p_{i j}\right) \leq 1$.

(iv) From the proof of (iii.), for each $k_{i} \in K_{i}$, it is easily seen that $p_{i_{1} j} \leq \mathscr{L} p_{i_{2} j} \leq \mathscr{L} p(+)_{j}$. According to the condition (v.) of Theorem 3.3, the condition $p_{i_{1} j} \leq \mathscr{L} p_{i_{2} j} \leq \mathscr{L} p(+)_{j}$ implies $U_{G}\left(p_{i_{2} j}, p(+)_{j}\right) \leq\left(U_{G}\left(p_{i_{1} j}, p(+)_{j}\right)\right.$. Note that in the denominator of (4.8), one has $U_{G}\left(p(-)_{j}, p(+)_{j}\right)>0$ because of the assumption of $p(-)_{j} \neq p(+)_{j}$ for $k_{i} \in K_{i}$. Thus, it is easily proven that $R I\left(p_{i_{2} j}\right) \leq R I\left(p_{i_{1} j}\right)$ for $k_{i} \in K_{i}$.

The proof of condition (v) can be proven as similar to (iv).

Example 4.5 Take the set of candidate alternatives $D=\left\{d_{1}, d_{2}, d_{3}\right\}$, and the criteria set $K=\left\{k_{1}, k_{2}\right\}$, where $K_{i}=\left\{k_{1}\right\}$ and $K_{i i}=\left\{k_{2}\right\}$. The PF decision matrix can be given as follows:

$p=\left[p_{i j}\right]_{3 \times 2}=\left[\begin{array}{cc}(0.54,0.67) & (071,0.43) \\ (0.88,0.35) & (0.62,0.49) \\ (0.50,0.56) & (0.85,0.36)\end{array}\right]$

The displaced positive-ideal $\mathrm{PF}$ values are $p(+)_{1}=$ $(0.88,0.35)$ and $p(+)_{2}=(0.62,0.49)$. Further, the displaced negative-ideal PF values are $p(-)_{1}=(0.50,0.67)$ and $p(-)_{2}=(0.85,0.36)$. Using the BPFSS and from 3.1, compute the Hamming distance (generalized distance measure for $\lambda=1$ ), which takes the form

$$
\begin{aligned}
& U_{H}\left(p_{i j}, p(+)_{j}\right)=\frac{1}{5} \\
& \left\{\begin{array}{c}
{\left[\left|u_{\left(p_{i j}\right)}-u_{\left(p(+)_{j}\right)}\right|+\left|v_{\left(p_{i j}\right)}-v_{\left(p(+)_{j}\right)}\right|+\left|\pi_{\left(p_{i j}\right)}-\pi_{\left(p(+)_{j}\right)}\right|\right.} \\
\left.+\left|r_{\left(p_{i j}\right)}-r_{\left(p(+)_{j}\right)}\right|+\left|d_{\left(p_{i j}\right)}-d_{\left(p(+)_{j}\right)}\right| \mid\right]
\end{array}\right\}
\end{aligned}
$$

From (4.9), $\quad \Omega_{H}=\left(p(-)_{1}, p(+)_{1}\right)=0.58 \quad$ and $\Omega_{H}=\left(p(-)_{2}, p(+)_{2}\right)=0.62 . \quad R I\left(p_{11}\right)=0.1205$, $R I\left(p_{12}\right)=0.176, \quad R I\left(p_{21}\right)=0.101, \quad R I\left(p_{22}\right)=0.13$, $R I\left(p_{31}\right)=0.155, R I\left(p_{32}\right)=0.127$.

Definition 4.6 Let $p_{i j}, p(\widehat{+})_{j}, p(\widehat{=})_{j}$ denote an assessment rating, the fixed PIPF value, and the fixed NIPF value, respectively, in the PF decision matrix $p=\left[p_{i j}\right]_{(m \times n)}$. The fixed RI $\widehat{R I}\left(p_{i j}\right)$ of $p_{i j}$ based on the generalized distance measure $U_{G}$ is defined as follows:

$$
\widehat{R I}\left(p_{i j}\right)=\frac{U_{G}\left(p_{i j}, p(\widehat{+})_{j}\right)}{U_{G}\left(p(\widehat{-})_{j}, p(\hat{+})_{j}\right)}
$$


Theorem 4.7 Choose the three assessment ratings $p_{i j}, p_{i_{1} j}, p_{i_{2} j}$. The fixed remoteness index satisfies the following properties:

i. $\widehat{R I}\left(p_{i j}\right)=0 \Leftrightarrow p_{i j}=p(\widehat{+})_{j} ;$

ii. $\widehat{R I}\left(p_{i j}\right)=1 \Leftrightarrow p_{i j}=p(\widehat{=})_{j}$;

iii. $0 \leq R I(\widehat{d})\left(p_{i j}\right) \leq 1$;

iv. For each $k_{j} \in K_{i}, \widehat{R I}\left(p_{i_{2} j}\right) \leq \widehat{R I}\left(p_{i_{1} j}\right)$, if $p_{i_{1} j} \leq \mathscr{L} p_{i_{2} j}$;

v. For each $k_{j} \in K_{i i}, \widehat{R I}\left(p_{i_{1} j}\right) \leq \widehat{R I}\left(p_{i_{2} j}\right)$, if $p_{i_{1} j} \leq \mathscr{L} p_{i_{2} j}$.

This theorem can be proved similar to Theorem 4.4.

Example 4.8 Let $D, K$ and $p=p_{i j}$ be as in Example 4.5. From Definition 4.2, the fixed positive-ideal PF values are $p(\widehat{+})_{1}=(1,0)$ and $p(\widehat{+})_{2}=(0,1)$. In the same way, the fixed negative-ideal PF values $p(\widehat{=})_{1}=(0,1)$ and $p(\widehat{=})_{2}=(1,0) . \quad$ Take $\lambda=1 . \quad$ Then, $\quad \Omega_{H}=\left(p(\widehat{-})_{1}\right.$, $\left.p(\widehat{+})_{1}\right)=\left(p(\widehat{二})_{2}, p(\widehat{+})_{2}\right)=0.6 . \quad \widehat{R I}\left(p_{11}\right)=0.1033$, $\widehat{R I}\left(p_{12}\right)=0.152, \quad \widehat{R I}\left(p_{21}\right)=0.098, \quad \widehat{R I}\left(p_{22}\right)=0.14$, $\widehat{R I}\left(p_{31}\right)=0.1035, \widehat{R I}\left(p_{32}\right)=0.15$.

As seen in this example, the oppositions between the remoteness indices become less discernible when anchoring subjective judgments with fixed ideal PF values. This situation is not so noticeable in Example 4.5.

\subsection{Precise and PF importance weights}

When decision-making in life, not all of the characteristics that affect these decisions are evaluated equally. Therefore, decision-making is determining alternatives according to the preferences and values of the decision-maker and choosing among them. Each situation has a weight for this choice. In DM operates, weights are assigned to the features. Two types of weights will be used in this study:

\section{Example 4.9}

(i) The precise importance weights are obtained by expectation score function. The weight vector $\omega$ calculated with $\frac{\mathscr{E} \mathscr{S}_{f(z)}}{\sum_{z} \mathscr{E} \mathscr{S}_{f}(z)}$.

Choose $f=$
$\left\{\left(k_{1}, 0.68,0.33\right),\left(k_{2}, 0.75,0.28\right),(k 3,0,84,0.39)\right\}$ $\left\{\left(k_{1}, 0.68,0.33\right),\left(k_{2}, 0.75,0.28\right),(k 3,0,84,0.39)\right\}$. Using the (2.9), $\mathscr{E} \mathscr{S}_{f\left(d_{1}\right)}=0.21435, \quad \mathscr{E} \mathscr{S}_{f\left(d_{2}\right)}$ $=0.17955, \mathscr{E} \mathscr{S}_{f\left(d_{3}\right)}=0.07115$ and $\sum_{i=1}^{3} \mathscr{E} \mathscr{S}_{f\left(d_{i}\right)}$ $=0.46505$. Then the weights are $\omega_{1}=0.461, \omega_{2}=$ 0.386 and $\omega_{3}=0.153\left(\sum_{i=1}^{3} \omega_{i}=1\right)$.

(ii) The PF Importance Weights: The PF importance weights of criteria $e_{j} \in \Sigma$ are displaced as the PFV $\omega_{j}=\left(\bar{\omega}_{j}, \underline{\omega}_{j}\right)$, where $\bar{\omega}_{j}$ and $\underline{\omega}_{j}$ represent the importance and unimportance degrees of the criteria $e_{j} \in \Sigma$, respectively. Throughout the DM process, the expert group examines the steps of the process and records their evaluations as a parametric Pythagorean fuzzy set (PPFS) at the end of the process. These PPFS built by experts will be used as PF importance weights in the DM process.

\subsection{Remoteness index-based new VIKOR methods}

New multiple criteria ranking indices for $R I\left(p_{i j}\right)$ and $\widehat{R I}\left(p_{i j}\right)$ measures of remoteness to the displaced and fixed ideal PF values, respectively, will be offered. In this way, the remoteness index-based new VIKOR method will be obtained. These multiple criteria ranking indices are: remoteness-based group utility indices, individual regret indices, and compromise indices. They can provide a mechanism to trade off a maximum group utility for the majority and a minimum individual regret for the opponent and can be used as an aggregating function for a compromise ranking among alternatives.

The smaller the value obtained in the displaced distance index $R I\left(p_{i j}\right)$, the more priority the assessment rating of the $p_{i j}$ will be. That is, the criterion-wise priority relationship among alternatives can be ordinarily identified according to ascending order of each $R I\left(p_{i j}\right)$ value. This is because displaced ideal PF values are to facilitate anchored judgments. In this study, a displaced remoteness-based compromise index by combining the suggested new definitions of a group utility index and an individual regret index is offered. The reason for this is to synthesize criteria-based priority relationships to obtain a compromise ranking on all criteria.

Definition 4.10 The displaced remoteness-based group utility index, the displaced remoteness-based individual regret index and the displaced remoteness-based compromise index are defined as follows:

$$
\begin{aligned}
\widetilde{\mathbb{S}}\left(d_{i}\right)= & \sum_{j=1}^{n}\left(R I\left(p_{i j}\right) \cdot \omega_{j}\right),\left(k_{j} \in K\right), \\
\widetilde{\mathbb{R}}\left(d_{i}\right)= & \max _{j=1}^{n}\left(R I\left(p_{i j}\right) \cdot \omega_{j}\right),\left(k_{j} \in K\right), \\
\widetilde{\mathbb{Q}}\left(d_{i}\right)= & \delta \cdot \frac{\mathbb{S}\left(d_{i}\right)-\min _{i^{\prime}=1}^{m} \mathbb{S}\left(d_{i^{\prime}}\right)}{\max _{i^{\prime}=1}^{m} \mathbb{S}\left(d_{i^{\prime}}\right)-\min _{i^{\prime}=1}^{m} \mathbb{S}\left(d_{i^{\prime}}\right)} \\
& +(1-\delta) \cdot \frac{\mathbb{R}\left(d_{i}\right)-\min _{i^{\prime}=1}^{m} \mathbb{R}\left(d_{i^{\prime}}\right)}{\max _{i^{\prime}=1}^{m} \mathbb{R}\left(d_{i^{\prime}}\right)-\min _{i^{\prime}=1}^{m} \mathbb{R}\left(d_{i^{\prime}}\right)}
\end{aligned}
$$

where $p=\left[p_{i j}\right]_{m \times n}$ and $\omega_{j}$ are PF decision matrix and a set of precise importance weights, respectively, and $\delta \in[0,1]$. 
Further, the conditions $0 \leq \omega_{j} \leq 1$ and $\sum_{j=1}^{n} \omega_{j}=1$ are the normalization conditions of precise weights.

Theorem 4.11 The ranking indices $\widetilde{\mathbb{S}}\left(d_{i}\right), \widetilde{\mathbb{R}}\left(d_{i}\right), \widetilde{\mathbb{Q}}\left(d_{i}\right)$ satisfy the following properties:

i. $\quad 0 \leq \widetilde{\mathfrak{S}}\left(d_{i}\right) \leq 1,0 \leq \widetilde{\mathbb{R}}\left(d_{i}\right) \leq 1$ and $0 \leq \widetilde{\mathbb{Q}}\left(d_{i}\right) \leq 1$;

ii. If $p_{i_{2} j} \leq \mathscr{L} p_{i_{1} j}$ and $p_{i_{1} j} \leq \mathscr{L} p_{i_{2} j}$ for all $k_{i} \in K_{i}$ and $k_{i} \in K_{i i}$, then $\widetilde{\mathbb{S}}\left(d_{i_{1}}\right) \leq \widetilde{\mathbb{S}}\left(d_{i_{2}}\right), \widetilde{\mathbb{R}}\left(d_{i_{1}}\right) \leq \widetilde{\mathbb{R}}\left(d_{i_{2}}\right)$ and $\widetilde{\mathbb{Q}}\left(d_{i_{1}}\right) \leq \widetilde{\mathbb{Q}}\left(d_{i_{2}}\right)$, respectively.

\section{Proof}

(i) Since $0 \leq R I\left(p_{i j}\right) \leq 1$ (the condition of (iii) of Theorem 4.4) and normalization conditions of precise weights, $0 \leq \widetilde{\mathbb{S}}\left(d_{i}\right) \leq 1,0 \leq \widetilde{\mathbb{R}}\left(d_{i}\right) \leq 1$ hold. Additionally, since $\widetilde{\mathbb{Q}}\left(d_{i}\right)$ is merely a linear combination of the normalized values of $\widetilde{S}\left(d_{i}\right), \widetilde{\mathbb{R}}\left(d_{i}\right)$, one can easily infer that $0 \leq \widetilde{\mathbb{Q}}\left(d_{i}\right) \leq 1$.

(ii) From the condition of (iv) of Theorem 4.4, since $p_{i_{2} j} \leq \mathscr{L} p_{i_{1} j}$ for each $k_{i} \in K_{i}$, then $\mathbb{R}\left(p_{i_{1} j}\right) \leq \mathbb{R}\left(p_{i_{2} j}\right)$. Since $p_{i_{1} j} \leq \mathscr{L} p_{i_{2} j}$ for each $k_{i} \in K_{i i}, \mathbb{R}\left(p_{i_{1} j}\right) \leq \mathbb{R}\left(p_{i_{2} j}\right)$ holds from the condition (v) of Theorem 4.4. That is, $\mathbb{R}\left(p_{i_{1} j}\right) \leq \mathbb{R}\left(p_{i_{2} j}\right)$ is fulfilled for $k_{i} \in K$. So, the inequalities $\widetilde{\mathbb{S}}\left(d_{i_{1}}\right) \leq \widetilde{\mathbb{S}}\left(d_{i_{2}}\right)$ and $\widetilde{\mathbb{R}}\left(d_{i_{1}}\right) \leq \widetilde{\mathbb{R}}\left(d_{i_{2}}\right)$ and $\widetilde{\mathbb{Q}}\left(d_{i_{1}}\right) \leq \widetilde{\mathbb{Q}}\left(d_{i_{2}}\right)$ are valid.

Next, consider an MCDA problem involving the PF decision matrix and PF importance weights. In a similar manner, this paper defines the multiple criteria ranking indices $\mathbb{S}\left(d_{i}\right), \mathbb{R}\left(d_{i}\right), \mathbb{Q}\left(d_{i}\right)$ under the condition of $\mathrm{PF}$ preference information. Analogous to Definition 4.10 , the product of $R I\left(p_{i j}\right)$ multiplied by $\omega_{j}$ must be calculated before determining these multiple criteria ranking indices. However, a difficulty in comparing the multiple criteria ranking indices among alternatives is encountered because $R I\left(p_{i j}\right) . \omega_{j} \mathrm{~s}$ a PF value when the PF importance weight $\omega_{j}$ is taken into account. Here, a score function approach to acquire a comparable value of $R I\left(p_{i j}\right) . \omega_{j}$ and then to identify the multiple criteria ranking indices is offered. It is worth noting that the score function approach leads to different ranges among $\mathbb{S}\left(d_{i}\right), \mathbb{R}\left(d_{i}\right), \mathbb{Q}\left(d_{i}\right)$ because the score function defined as in (2.4) is between -1 and 1 .

Definition 4.12 The displaced remoteness-based group utility index, the displaced remoteness-based individual regret index and the displaced remoteness-based compromise index are defined as follows:

$$
\begin{aligned}
\mathbb{S}\left(d_{i}\right)= & \sum_{j=1}^{n} \mathscr{E} \mathscr{S}\left(R I\left(p_{i j}\right) \cdot \omega_{j}\right) \\
= & \sum_{j=1}^{n}\left\{\frac{1}{2}\left[2-\left(1-\left(\underline{\omega}_{j}\right)^{2}\right)^{R I\left(p_{i j}\right)}-\left(\bar{\omega}_{j}\right)^{2 \cdot R I\left(p_{i j}\right)}\right]\right\} \\
& \left(k_{j} \in K\right), \\
\mathbb{R}\left(d_{i}\right)= & \max _{j=1}^{n} \mathscr{E} \mathscr{S}\left(R I\left(p_{i j}\right) \cdot \omega_{j}\right) \\
= & \max _{j=1}^{n}\left\{\frac{1}{2}\left[2-\left(1-\left(\underline{\omega}_{j}\right)^{2}\right)^{R I\left(p_{i j}\right)}-\left(\bar{\omega}_{j}\right)^{2 \cdot R I\left(p_{i j}\right)}\right]\right\} \\
& \left(k_{j} \in K\right), \\
\mathbb{Q}\left(d_{i}\right)= & \delta \cdot \frac{\mathbb{S}\left(d_{i}\right)-\min _{i^{\prime}=1}^{m} \mathbb{S}\left(d_{i^{\prime}}\right)}{\max _{i^{\prime}=1}^{m} \mathbb{S}\left(d_{i^{\prime}}\right)-\min _{i^{\prime}=1}^{m} \mathbb{S}\left(d_{i^{\prime}}\right)} \\
& +(1-\delta) \cdot \frac{\mathbb{R}\left(d_{i}\right)-\min _{i^{\prime}=1}^{m} \mathbb{R}\left(d_{i^{\prime}}\right)}{\max _{i^{\prime}=1}^{m} \mathbb{R}\left(d_{i^{\prime}}\right)-\min _{i^{\prime}=1}^{m} \mathbb{R}\left(d_{i^{\prime}}\right)}
\end{aligned}
$$

where $p=\left[p_{i j}\right]_{m \times n}$ and $\omega_{j}$ are PF decision matrix and a set of PF importance weights, respectively, and $\delta \in[0,1]$.

Theorem 4.13 The ranking indices $\mathbb{S}\left(d_{i}\right), \mathbb{R}\left(d_{i}\right), \mathbb{Q}\left(d_{i}\right)$ satisfy the following properties:

i. $\quad-n \leq \mathbb{S}\left(d_{i}\right) \leq n,-1 \leq \mathbb{R}\left(d_{i}\right) \leq 1$ and $0 \leq \mathbb{Q}\left(d_{i}\right) \leq 1 ;$

ii. If $p_{i_{2} j} \leq \mathscr{L} p_{i_{1} j}$ and $p_{i_{1} j} \leq \mathscr{L} p_{i_{2} j}$ for all $k_{i} \in K_{i}$ and $k_{i} \in K_{i i}$, then $\mathbb{S}\left(d_{i_{1}}\right) \leq \mathbb{S}\left(d_{i_{2}}\right), \mathbb{R}\left(d_{i_{1}}\right) \leq \mathbb{R}\left(d_{i_{2}}\right)$ and $\mathbb{Q}\left(d_{i_{1}}\right) \leq \mathbb{Q}\left(d_{i_{2}}\right)$, respectively.

Proof According to the (2.9), the inequality $-1 \leq \mathscr{E} \mathscr{S}\left(R I\left(p_{i j}\right) \cdot \omega_{j}\right) \leq 1 \quad\left(\forall k_{j} \in K\right) \quad$ holds. Then, $-1 \leq \max _{j=1}^{n} \mathscr{E} \mathscr{S}\left(R I\left(p_{i j}\right) . \omega_{j}\right) \leq 1$ is valid. From here, $-1 \leq \mathbb{R}\left(d_{i}\right) \leq 1$ is obtained. At the same time, $-n \leq \sum_{j=1}^{n} \mathscr{E} \mathscr{S}\left(R I\left(p_{i j}\right) . \omega_{j}\right) \leq n \Rightarrow-n \leq \mathbb{R}\left(d_{i}\right) \leq n$. Consider (4.16). Since $\mathbb{Q}\left(d_{i}\right)$ is merely a linear combination of the normalized values $\mathbb{S}\left(d_{i}\right)$, and $\mathbb{R}\left(d_{i}\right)$, then, it is obvious that it will be $0 \leq \mathbb{Q}\left(d_{i}\right) \leq 1$.

The proof of the condition (ii.) can be easily get from part (ii.) of Theorem 4.11

For fixed ideal PF values, new definitions are given as follows:

Definition 4.14 The fixed remoteness-based group utility index, the fixed remoteness-based individual regret index and the fixed remoteness-based compromise index are defined as follows: 


$$
\begin{aligned}
\widetilde{\mathbb{S F}}\left(d_{i}\right)= & \sum_{j=1}^{n}\left(\widehat{R I}\left(p_{i j}\right) \cdot \omega_{j}\right),\left(k_{j} \in K\right), \\
\widetilde{\mathbb{R} F}\left(d_{i}\right)= & \max _{j=1}^{n}\left(\widehat{R I}\left(p_{i j}\right) \cdot \omega_{j}\right),\left(k_{j} \in K\right), \\
\widetilde{\mathbb{Q} F}\left(d_{i}\right)= & \delta \cdot \frac{\widetilde{\mathbb{S F}}\left(d_{i}\right)-\min _{i^{\prime}=1}^{m} \widetilde{\mathbb{S F}}\left(d_{i^{\prime}}\right)}{\max _{i^{\prime}=1}^{m} \widetilde{\mathbb{S F}}\left(d_{i^{\prime}}\right)-\min _{i^{\prime}=1}^{m} \widetilde{\mathbb{S F}}\left(d_{i^{\prime}}\right)} \\
& +(1-\delta) \cdot \frac{\widetilde{\mathbb{R F}}\left(d_{i}\right)-\min _{i^{\prime}=1}^{m} \widetilde{\mathbb{R F}}\left(d_{i^{\prime}}\right)}{\max _{i^{\prime}=1}^{m} \widetilde{\mathbb{R F}}\left(d_{i^{\prime}}\right)-\min _{i^{\prime}=1}^{m} \widetilde{\mathbb{R F}}\left(d_{i^{\prime}}\right)}
\end{aligned}
$$

where $p=\left[p_{i j}\right]_{m \times n}$ and $\omega_{j}$ are PF decision matrix and a set of precise importance weights, respectively, and $\delta \in[0,1]$. Further, the conditions $0 \leq \omega_{j} \leq 1$ and $\sum_{j=1}^{n} \omega_{j}=1$ are the normalization conditions of precise weights.

Definition 4.15 The fixed remoteness-based group utility index, the fixed remoteness-based individual regret index and the fixed remoteness-based compromise index are defined as follows:

$$
\begin{aligned}
\mathbb{S F}\left(d_{i}\right) & =\sum_{j=1}^{n} \mathscr{E} \mathscr{S}\left(\widehat{R I}\left(p_{i j}\right) \cdot \omega_{j}\right) \\
& =\sum_{j=1}^{n}\left\{\frac{1}{2}\left[2-\left(1-\left(\underline{\omega}_{j}\right)^{2}\right)^{\widehat{R I}\left(p_{i j}\right)}-\left(\bar{\omega}_{j}\right)^{2 \cdot \widehat{R I}\left(p_{i j}\right)}\right]\right\}, \\
& \left(k_{j} \in K\right)
\end{aligned}
$$

$$
\begin{aligned}
\mathbb{R F}\left(d_{i}\right)= & \max _{j=1}^{n} \mathscr{E} \mathscr{S}\left(\widehat{R I}\left(p_{i j}\right) \cdot \omega_{j}\right) \\
= & \max _{j=1}^{n}\left\{\frac{1}{2}\left[2-\left(1-\left(\underline{\omega}_{j}\right)^{2}\right)^{\widehat{R I}\left(p_{i j}\right)}-\left(\bar{\omega}_{j}\right)^{2 . \widehat{R I}\left(p_{i j}\right)}\right]\right\}, \\
& \left(k_{j} \in K\right),
\end{aligned}
$$

$$
\begin{aligned}
& \mathbb{Q} \mathbb{F}\left(d_{i}\right)=\delta \cdot \frac{\mathbb{S F}\left(d_{i}\right)-\min _{i^{\prime}=1}^{m} \operatorname{SF}\left(d_{i^{\prime}}\right)}{\max _{i^{\prime}=1}^{m} \operatorname{SF}\left(d_{i^{\prime}}\right)-\min _{i^{\prime}=1}^{m} \operatorname{SF}\left(d_{i^{\prime}}\right)} \\
&+(1-\delta) \cdot \frac{\mathbb{R F}\left(d_{i}\right)-\min _{i^{\prime}=1}^{m} \mathbb{R} \mathbb{F}\left(d_{i^{\prime}}\right)}{\max _{i^{\prime}=1}^{m} \mathbb{R} \mathbb{F}\left(d_{i^{\prime}}\right)-\min _{i^{\prime}=1}^{m} \mathbb{R F}\left(d_{i^{\prime}}\right)}
\end{aligned}
$$

where $p=\left[p_{i j}\right]_{m \times n}$ and $\omega_{j}$ are PF decision matrix and a set of PF importance weights, respectively, and $\delta \in[0,1]$.

Theorem $\quad 4.16$ The ranking indices $\widetilde{\mathbb{S F}}\left(d_{i}\right), \widetilde{\mathbb{R F}}\left(d_{i}\right), \widetilde{\mathbb{Q} F}\left(d_{i}\right)$ satisfy the following properties:

i. $0 \leq \widetilde{\mathbb{S F}}\left(d_{i}\right) \leq 1,0 \leq \widetilde{\mathbb{R F}}\left(d_{i}\right) \leq 1$ and $0 \leq \widetilde{\mathbb{Q F}}\left(d_{i}\right) \leq 1$; ii. If $p_{i_{2} j} \leq \mathscr{L} p_{i_{1} j}$ and $p_{i_{1} j} \leq \mathscr{L} p_{i_{2} j}$ for all $k_{i} \in K_{i}$ and $k_{i} \in K_{i i}$, then $\widetilde{\mathbb{S F}}\left(d_{i_{1}}\right) \leq \widetilde{\mathbb{S F}}\left(d_{i_{2}}\right), \widetilde{\mathbb{R F}}\left(d_{i_{1}}\right) \leq \widetilde{\mathbb{R F}}\left(d_{i_{2}}\right)$ and $\widetilde{\mathbb{Q F}}\left(d_{i_{1}}\right) \leq \widetilde{\mathbb{Q F}}\left(d_{i_{2}}\right)$, respectively.

Theorem 4.17 The ranking indices $\mathbb{S} \mathbb{F}\left(d_{i}\right), \mathbb{R F}\left(d_{i}\right), \mathbb{Q} \mathbb{F}\left(d_{i}\right)$ satisfy the following properties:

$$
\begin{array}{ll}
\text { i. } & -n \leq \mathbb{S} \mathbb{F}\left(d_{i}\right) \leq n, \quad-1 \leq \mathbb{R} \mathbb{F}\left(d_{i}\right) \leq 1 \quad \text { and } \\
& 0 \leq \mathbb{Q} \mathbb{F}\left(d_{i}\right) \leq 1 ; \\
\text { ii. } & \text { If } p_{i_{2} j} \leq \mathscr{L} p_{i_{1} j} \text { and } p_{i_{1} j} \leq \mathscr{L} p_{i_{2} j} \text { for all } k_{i} \in K_{i} \text { and } \\
& k_{i} \in K_{i i}, \text { then } \mathbb{S} \mathbb{F}\left(d_{i_{1}}\right) \leq \mathbb{S} \mathbb{F}\left(d_{i_{2}}\right), \mathbb{R} \mathbb{F}\left(d_{i_{1}}\right) \leq \mathbb{R} \mathbb{F}\left(d_{i_{2}}\right) \\
& \text { and } \mathbb{Q} \mathbb{F}\left(d_{i_{1}}\right) \leq \mathbb{Q} \mathbb{F}\left(d_{i_{2}}\right) \text {, respectively. }
\end{array}
$$

These theorems can be proved as Theorems 4.11 and 4.13.

The decision process coefficient is shown by $\delta$. One can modify decision making strategy by changing the value of $\delta$. The value of parameter $\delta$ indicates the importance of maximum group utility, while $1-\delta$ indicates the importance of individual regrets. In the classical VIKOR method, the higher the value of $\delta$ (when $\delta>0.5$ ), the compromise ranking procedure is categorized as the procedure with voting by majority. The compromise ranking procedure is categorized as the procedure with veto when $\delta<0.5$. The consensus is achieved in the compromise ranking procedure at $\delta=0.5$.

Example 4.18 Consider the $(F, M, f)$ of Example 3.5. Here, Let $M_{1}=\left\{e_{1}, e_{2}\right\}$ and $M_{2}=\left\{e_{3}\right\}\left(M=M_{1} \cup M_{2}\right)$. For $\delta=0.5$ and $\lambda=1$,

(i). Using the expectation score function, calculate the precise importance weights: $\omega_{1}=0.158811626$, $\omega_{2}=0.493797108, \omega_{3}=0.347391266$. Choose $\lambda=1$ and $\delta=0.5$. From Definition 4.10, results are obtained: $\quad R I\left(p_{11}\right)=0, \quad R I\left(p_{12}\right)=0$, $R I\left(p_{13}\right)=0.911, \quad R I\left(p_{21}\right)=0.0406$, $R I\left(p_{22}\right)=0.98, R I\left(p_{23}\right)=0$ and

$$
\begin{gathered}
\widetilde{S}\left(x_{1}\right)=R I\left(p_{11}\right) \cdot \omega_{1}+R I\left(p_{12}\right) \cdot \omega_{2}+R I\left(p_{13}\right) . \\
\omega_{3}=0.32, \\
\widetilde{S}\left(x_{2}\right)=R I\left(p_{21}\right) \cdot \omega_{1}+R I\left(p_{22}\right) \cdot \omega_{2}+R I\left(p_{23}\right) . \\
\omega_{3}=0.659
\end{gathered}
$$

and $\widetilde{\mathbb{R}}\left(x_{1}\right)=0.32, \quad \widetilde{\mathbb{R}}\left(x_{2}\right)=0.484$. Then, $\widetilde{\mathbb{Q}}\left(x_{1}\right)=0$ and $\widetilde{\mathbb{Q}}\left(x_{2}\right)=1$. Therefore, the rankings are obtained: $x_{1}<x_{2}$ for $\widetilde{\mathbb{S}}\left(x_{i}\right), x_{1}<x_{2}$ for $\widetilde{\mathbb{R}}\left(x_{i}\right)$, and $x_{1}<x_{2}$ for $\widetilde{\mathbb{Q}}\left(x_{i}\right)$. Similar calculations can be made for Definition 4.14. 
(ii). The PF importance weights are given as $\omega_{1}=$ $\left(\underline{\omega_{1}}, \overline{\omega_{1}}\right)=(0.65,0.35)$ and $\omega_{2}=(0.55,0.50)$, $\widehat{\omega_{3}}=(0.60,0.60)$. From Definition 4.12, results are obtained: $\tilde{S}\left(x_{1}\right)=0.467, \quad \tilde{S}\left(x_{2}\right)=1.195$, $\widetilde{R}\left(x_{1}\right)=0.467, \quad \widetilde{R}\left(x_{2}\right)=0.675$ and $\widetilde{Q}\left(x_{1}\right)=0$, $\widetilde{Q}\left(x_{2}\right)=1$. Therefore, the rankings are obtained: $x_{1}<x_{2}$ for $\widetilde{S}\left(x_{i}\right), x_{1}<x_{2}$ for $\widetilde{R}\left(x_{i}\right)$, and $x_{1}<x_{2}$ for $\widetilde{Q}\left(x_{i}\right)$. Similar calculations can be made for Definition 4.15 .

\section{Quarantine situations application}

In this section, firstly, using newly defined distance measurements, weights, displaced and fixed ideals, and displaced and fixed remoteness indices novel algorithms will be given. Later, these algorithms will be applied to the cognitive and behavioral development of early childhood children staying at home in the COVID-19 quarantine.

\subsection{Algorithms}

For the four different scenarios, algorithms are given as follows:

Algorithm 1 PF preference information, anchored judgments with DIs

S 1: Choose the set of alternatives $D=\left\{d_{1}, d_{2}, \cdots, d_{n}\right\}$ and the set of criteria $K=\left\{k_{1}, k_{2}, \cdots, k_{m}\right\}$ (divided into $K_{1}$ and $K_{2}$ ).

S 2: Give the PFV for each alternative, compute the PF importance weights $\omega_{j}$ and establish the PF assessment rating for each $d_{i} \in D$ with respect $k i \in K$. Identify the PF decision matrix $p=\left[p_{i j}\right]_{m \times n}$.

S 3: Find the displaced PIPF value $p(+)_{j}$ (Use 4.2) and the displaced NIPF value $p(-)_{j}$ (Use 4.3) with respect to each criterion $k_{j} \in K$.

S 4: For $1 \leq i \leq m$ and $1 \leq j \leq n$, calculate $U_{G}\left(p(-)_{j}, p(+)_{j}\right)$ and $U_{G}\left(p_{i j}, p(+)_{j}\right)$, (Apply 3.2).

S 5: For each $d_{i} \in D$ and $k_{j} \in K$, calculate displaced RI $R I\left(p_{i j}\right)$, (Employ 4.8).

S 6: Compute the index $\widetilde{\mathbb{S}}\left(d_{i}\right)$ and the index $\widetilde{\mathbb{R}}\left(d_{i}\right)$ (Use 4.14 and 4.15).

S 7: After setting the parameter $\delta$ as a coefficient of the decision process, obtain the index $\widetilde{\mathbb{Q}}\left(d_{i}\right),(0 \leq \delta \leq 1)($ Apply 4.16).

S 8: Three ranking lists obtained by sorting the values $\widetilde{\mathbb{S}}\left(d_{i}\right), \widetilde{\mathbb{R}}\left(d_{i}\right), \widetilde{\mathbb{Q}}\left(d_{i}\right)$ for each alternative in ascending order.

S 9: While $D^{(1)} \subseteq D$ and

$$
D^{(1)}=\left\{d_{i}: \min _{i=1}^{m} \widetilde{\mathbb{Q}}\left(d_{i}\right)\right\}
$$

recommend the alternative(or alternatives) $d^{(1)} \in D^{(1)}$ such that the alternative(or alternatives) in $\widetilde{\mathbb{Q}}\left(d_{i}\right)$ ranking list is (are) the ultimate compromise solution if the cases (a) and (b) are both satisfied:

Case (a) For $D^{(2)} \subseteq D$ and $d^{(2)} \in D^{(2)}$,

$$
\widetilde{\mathbb{Q}}\left(d^{(2)}\right)-\widetilde{\mathbb{Q}}\left(d^{(1)}\right) \geq \frac{1}{m-1}
$$

is (are) the alternative (or alternatives) in the second position in the list ranked by $\widetilde{\mathbb{Q}}\left(d_{i}\right)$.

This case is called the assessment of acceptable advantage.

Case $(b) d^{(1)}$ must also be the best ranked by $\widetilde{\mathbb{S}}\left(d_{i}\right)$ and $\widetilde{\mathbb{R}}\left(d_{i}\right)$.

This case is called the assessment of acceptable stability in decision-making.

When one of these conditions is not met, the following two conditions will apply to the set of ultimate compromise solutions:

(i.) If the case (b) is not satisfied, then the set of ultimate compromise solutions is consist of the alternatives $d^{(1)}$ and $d^{(2)}$;

(ii.) If the case (a) is not satisfied, then the set of ultimate compromise solutions is consist of the alternatives $d^{(1)}, d^{(2)}, \cdots d^{(r)}$ such that

$$
\widetilde{\mathbb{Q}}\left(d^{(r)}\right)-\widetilde{\mathbb{Q}}\left(d^{(1)}\right)<\frac{1}{m-1},
$$

where $r$ is the largest $i$. This inequality implies that the positions $d^{(1)}, d^{(2)}, \cdots d^{(r)}$ are in closeness. 
Algorithm 2 PF preference information, anchored judgments with FIs

S 1: Choose the set of alternatives $D=\left\{d_{1}, d_{2}, \cdots, d_{n}\right\}$ and the set of criteria $K=\left\{k_{1}, k_{2}, \cdots, k_{m}\right\}$ (divided into $K_{1}$ and $K_{2}$ ).

S 2: Give the PFV for each alternative, compute the PF importance weights $\omega_{j}$ and establish the PF assessment rating for each $d_{i} \in D$ with respect $k i \in K$. Identify the PF decision matrix $p=\left[p_{i j}\right]_{m \times n}$.

S 3: Find the fixed PIPF value $p(\widehat{+})_{j}$ (Use 4.6)and the fixed NIPF value $p(\widehat{-})_{j}$ (Use 4.7) with respect to each criterion $k_{j} \in K$.

S 4: Calculate $U_{G}\left(p(\widehat{-})_{j}, p(\widehat{+})_{j}\right)$ and $U_{G}\left(p_{i j}, p(\widehat{+})_{j}\right),(1 \leq i \leq m$ and $1 \leq j \leq n)$ (Apply 3.2).

S 5: Calculate fixed RI $\widehat{R} I\left(p_{i j}\right)$, (for each $d_{i} \in D$ and $k_{j} \in K$ ) (Employ 4.10).

S 6: Compute the index $\mathbb{S F}\left(d_{i}\right)$ and the index $\operatorname{RF}\left(d_{i}\right)$ (Use 4.20 and 4.21).

S 7: After setting the parameter $\delta$ as a coefficient of the decision process, obtain the index $\mathbb{Q F}\left(d_{i}\right),(0 \leq \delta \leq 1)($ Apply 4.22$)$.

S 8: Three ranking lists obtained by sorting the values $\mathbb{S F}\left(d_{i}\right), \mathbb{R F}\left(d_{i}\right), \mathbb{Q F}\left(d_{i}\right)$ for each alternative in ascending order.

S 9: While $D^{(1)} \subseteq D$ and

$$
D^{(1)}=\left\{d_{i}: \min _{i=1}^{m} \mathrm{QF}\left(d_{i}\right)\right\}
$$

recommend the alternative(or alternatives) $d^{(1)} \in D^{(1)}$ such that the alternative(or alternatives) in $\mathbb{Q F}\left(d_{i}\right)$ ranking list is (are) the ultimate compromise solution if the cases (a) and (b) are both satisfied:

Case (a) For $D^{(2)} \subseteq D$ and $d^{(2)} \in D^{(2)}$

$$
\mathbb{Q F}\left(d^{(2)}\right)-\mathbb{Q F}\left(d^{(1)}\right) \geq \frac{1}{m-1} .
$$

Case (b) $d^{(1)}$ must also be the best ranked by $\operatorname{SF}\left(d_{i}\right)$ and $\mathbb{R F}\left(d_{i}\right)$. When one of these conditions is not met, the following two conditions will apply to the set of ultimate compromise solutions:

(i.) If the case (b) is not satisfied, then the set of ultimate compromise solutions is consist of the alternatives $d^{(1)}$ and $d^{(2)}$;

(ii.) If the case (a) is not satisfied, then the set of ultimate compromise solutions is consist of the alternatives $d^{(1)}, d^{(2)}, \cdots d^{(r)}$ such that

$$
\mathbb{Q F}\left(d^{(r)}\right)-\mathbb{Q F}\left(d^{(1)}\right)<\frac{1}{m-1} .
$$


Algorithm 3 Precise preference information, anchored judgments with DIs

S 1: Choose the set of alternatives $D=\left\{d_{1}, d_{2}, \cdots, d_{n}\right\}$ and the set of criteria $K=\left\{k_{1}, k_{2}, \cdots, k_{m}\right\}$ (divided into $K_{1}$ and $K_{2}$ ).

S 2: Give the PFV for each alternative, compute the precise importance weights $\omega_{j}$ and establish the PF assessment rating for each $d_{i} \in D$ with respect $k i \in K$. Identify the PF decision matrix $p=\left[p_{i j}\right]_{m \times n}$.

S 3: Find the displaced PIPF value $p(+)_{j}$ and the displaced NIPF value $p(-)_{j}$ with respect to each criterion $k_{j} \in K$.

S 4: Calculate $U_{G}\left(p(-)_{j}, p(+)_{j}\right)$ and $U_{G}\left(p_{i j}, p(+)_{j}\right),(1 \leq i \leq m$ and $1 \leq j \leq n)$.

S 5: Calculate displaced RI $R I\left(p_{i j}\right)$, (for each $d_{i} \in D$ and $k_{j} \in K$ ).

S 6: Compute the index $\widetilde{\mathbb{S}}\left(d_{i}\right)$ and the index $\widetilde{\mathbb{R}}\left(d_{i}\right)$ (Use 4.11 and 4.12 ).

S 7: After setting the parameter $\delta$ as a coefficient of the decision process, obtain the index $\widetilde{\mathbb{Q}}\left(d_{i}\right),(0 \leq \delta \leq 1)$ (Apply 4.13).

S 8: Three ranking lists obtained by sorting the values $\widetilde{\mathbb{S}}\left(d_{i}\right), \widetilde{\mathbb{R}}\left(d_{i}\right), \widetilde{\mathbb{Q}}\left(d_{i}\right)$ for each alternative in ascending order.

S 9: While $D^{(1)} \subseteq D$ and

$$
D^{(1)}=\left\{d_{i}: \min _{i=1}^{m} \widetilde{\mathbb{Q}}\left(d_{i}\right)\right\}
$$

recommend the alternative(or alternatives) $d^{(1)} \in D^{(1)}$ such that the alternative(or alternatives) in $\widetilde{\mathbb{Q}}\left(d_{i}\right)$ ranking list is (are) the ultimate compromise solution if the cases (a) and (b) are both satisfied:

Case (a) For $D^{(2)} \subseteq D$ and $d^{(2)} \in D^{(2)}$,

$$
\widetilde{\mathbb{Q}}\left(d^{(2)}\right)-\widetilde{\mathbb{Q}}\left(d^{(1)}\right) \geq \frac{1}{m-1}
$$

is (are) the alternative (or alternatives) in the second position in the list ranked by $\widetilde{\mathbb{Q}}\left(d_{i}\right)$.

This case is called the assessment of acceptable advantage.

Case (b) $d^{(1)}$ must also be the best ranked by $\widetilde{\mathbb{S}}\left(d_{i}\right)$ and $\widetilde{\mathbb{R}}\left(d_{i}\right)$.

This case is called the assessment of acceptable stability in decision-making. When one of these conditions is not met, the following two conditions will apply to the set of ultimate compromise solutions:

(i.) If the case (b) is not satisfied, then the set of ultimate compromise solutions is consist of the alternatives $d^{(1)}$ and $d^{(2)}$;

(ii.) If the case (a) is not satisfied, then the set of ultimate compromise solutions is consist of the alternatives $d^{(1)}, d^{(2)}, \cdots d^{(r)}$ such that

$$
\widetilde{\mathbb{Q}}\left(d^{(r)}\right)-\widetilde{\mathbb{Q}}\left(d^{(1)}\right)<\frac{1}{m-1} .
$$


Algorithm 4 Precise preference information, anchored judgments with FIs

S 1: Choose the set of alternatives $D=\left\{d_{1}, d_{2}, \cdots, d_{n}\right\}$ and the set of criteria $K=\left\{k_{1}, k_{2}, \cdots, k_{m}\right\}$ (divided into $K_{1}$ and $K_{2}$ ).

S 2: Give the PFV for each alternative, compute the precise importance weights $\omega_{j}$ and establish the PF assessment rating for each $d_{i} \in D$ with respect $k i \in K$. Identify the PF decision matrix $p=\left[p_{i j}\right]_{m \times n}$.

S 3: Find the fixed PIPF value $p(\widehat{+})_{j}$ and the fixed NIPF value $p(\widehat{-})_{j}$ with respect to each criterion $k_{j} \in K$.

S 4: Calculate $U_{G}\left(p(\widehat{-})_{j}, p(\widehat{+})_{j}\right)$ and $U_{G}\left(p_{i j}, p(\widehat{+})_{j}\right),(1 \leq i \leq m$ and $1 \leq j \leq n)$.

S 5: Calculate fixed RI $\widehat{R} I\left(p_{i j}\right)$, (for each $d_{i} \in D$ and $k_{j} \in K$ ).

S 6: Compute the index $\mathbb{S F}\left(d_{i}\right)$ and the index $\mathbb{R F}\left(d_{i}\right)$ (Use 4.17 and 4.18).

S 7: After setting the parameter $\delta$ as a coefficient of the decision process, obtain the index $\mathbb{Q F}\left(d_{i}\right),(0 \leq \delta \leq 1)($ Apply 4.19).

S 8: Three ranking lists obtained by sorting the values $\mathbb{S F}\left(d_{i}\right), \mathbb{R F}\left(d_{i}\right), \mathbb{Q F}\left(d_{i}\right)$ for each alternative in ascending order.

S 9: While $D^{(1)} \subseteq D$ and

$$
D^{(1)}=\left\{d_{i}: \min _{i=1}^{m} \mathrm{QF}\left(d_{i}\right)\right\}
$$

recommend the alternative(or alternatives) $d^{(1)} \in D^{(1)}$ such that the alternative(or alternatives) in $\mathbb{Q F}\left(d_{i}\right)$ ranking list is (are) the ultimate compromise solution if the cases (a) and (b) are both satisfied:

Case (a)

$$
\mathbb{Q F}\left(d^{(2)}\right)-\mathbb{Q F}\left(d^{(1)}\right) \geq \frac{1}{m-1}
$$

Case $(b) d^{(1)}$ must also be the best ranked by $\mathbb{S F}\left(d_{i}\right)$ and/or $\mathbb{R F}\left(d_{i}\right)$.

When one of these conditions is not met, the following two conditions will apply to the set of ultimate compromise solutions:

(i.) If the case (b) is not satisfied, then the set of ultimate compromise solutions is consist of the alternatives $d^{(1)}$ and $d^{(2)}$;

(ii.) If the case (a) is not satisfied, then the set of ultimate compromise solutions is consist of the alternatives $d^{(1)}, d^{(2)}, \cdots d^{(r)}$ such that

$$
\mathbb{Q F}\left(d^{(r)}\right)-\mathbb{Q F}\left(d^{(1)}\right)<\frac{1}{m-1} .
$$

\subsection{Quarantine application for early childhood children}

In natural disasters and especially in epidemic diseases, some measures are taken to protect people from the negative effects of the situation. One of the measures that can be taken is quarantine. The quarantine is used to indicate restrictions on the activities of people or animals exposed to infectious diseases during the infectious period. Children, who are members of society and cannot be isolated from society, should be informed correctly and sufficiently to prevent them from being affected by both the biological effect and the psychological effect of the epidemic. It is extremely important to inform and raise awareness of children beforehand in order to prevent a pandemic because children may face troubles due to the long duration of natural disasters and measures such as quarantine restricting people. Children may face personal losses, collective deaths, and discomfort caused by the diseases caught in natural disasters and outbreaks. These situations can cause adversities such as stress, anxiety, depression, and behavioral disorders in children.

Children learn a lot of the information they learn through environmental stimuli. The interaction of the child with his/her environment, social relationships, other people, especially adults, plays a very important role in cognitive development. The stimuli that it is exposed to in the pandemic process direct the perception of children to the pandemic. In this case, it is clear that children will pay more attention to the pandemic, quarantine, and related stimuli. In the process, the vast majority of stimuli around children, including parents and digital media, lead their perception of COVID-19. If this perception cannot be controlled properly, a false cognition and belief in children will be inevitable.

In this study, the survey model was used. The survey was prepared to be answered on the internet. Survey questions were asked to children aged 5-6 and their families. The linguistic terms and their numeric labels are: For Questions to be asked to the child: Yes(1), maybe/some (2), no(3). For Questions to be asked to parents: too much(1), much (2), some (3), too little(4), none (5).

The survey included the following questions:

Questions to be asked to the child:

- Do you know Corona-virus?

- Are you afraid of Corona-virus?

- Does Corona-virus harm people?

- Does Corona-virus harm animals?

- Can Corona-virus be prevented?

- Do you think it's nice not to go to school?

- Are you upset that you can't go to school? 
- Is the obligation to stay home boring?

- Can we be protected from Corona-virus by staying at home?

- Do you think you can go to school from now on?

Questions to be asked to parents:

- Does your child behave anxiously after Corona-virus?

- Is your child afraid when a conversation about Coronavirus has passed?

- Does your child ask about Corona-virus?

- Does your child pay more attention to cleaning after Corona-virus?

- Has your child's sleep pattern been impaired after Corona-virus?

- Have there been changes in your child's nutritional habits after Corona-virus?

- Did your child develop undesirable behaviour after Corona-virus?

- Is your child happy because she/he can't go to school?

- Has the time your child spent on the Internet after Corona-virus increased?

- Has the time your child spent in front of the TV increased after Corona-virus?

The cognitive and behavioural distributions of questions are as follows:

For children's cognition;

$c_{1}$ : Do children know about the current situation? (4 questions)

$c_{2}$ : Does the current situation affect children's emotions? (4 questions)

$c_{3}$ : Does the current situation affect children's thoughts? (2 questions)

For children's behavioural;

$d_{1}$ : Has Corona-virus changed the basic habits of children? (3 questions)

$d_{2}$ : Did behaviour change occur in children after quarantine? (5 questions)

$d_{3}$ : Did children's behaviour regarding information technologies increase after quarantine? (2 questions)

The target audience is children aged 5-6 in early childhood. Children of this age group are in the process of gaining skills in expressing their feelings during this period. In addition, the emotional responses of these children can be noticed by a careful observer or even an expert. This section is to investigate the effects of quarantine status due to the COVID-19 pandemic on the cognitions and behaviors of children who stay at home. For this, questions were asked to the children and their parents. Opinions of each child and each parent about the questions asked were got. The effect of quarantine on their own cognition in line with the answers given by the children and the effect of the behavior of their children in line with the observations of the parents have been revealed.

Let $C=\left\{c_{1}, c_{2}, c_{3}\right\}$ and $D=\left\{d_{1}, d_{2}, d_{3}\right\}$ represent the sets of the questions related to children's cognition and the questions related to children's behavioral, respectively. Let the sets $M=\left\{m_{1}, m_{2}, m_{3}\right\}=\{y e s$, maybe, no $\}$ and $N=$ $\left\{n_{1}, n_{2}, n_{3}\right\}=\{$ much, some, none $\}$ represent the answer given by the children to the questions asked and answer given by the parents to the questions asked, respectively.

An expert group was formed to ask questions to children and evaluate their answers. The experts assessed the answers according to criteria. The experts gave their judgments based on their knowledge and previous statistical measures. The committee preferences are stored in the form of GPFSS. These results are shown in Tables 2 and 3. $f$ and $g$ in these tables represent expert opinions. Algorithm-based solutions will be made for both cognitive and behavioral developments.

As demonstrated in Theorem 3.3 and Note 1, the Hamming distance $\Omega_{H}$ is a metric because of its reflexivity, symmetry, separability and triangle inequality. Thus, when computing the generalized distance measure $\Omega_{G}$, this paper sets the distance parameter $\lambda=1$ for the application. Moreover, assume that the decision-maker would like to achieve the goal of "consensus," and thus, set $\delta=0,5$.

Algorithm 1 for $(F, M, f)$ :

A simple MCDA problem concerning three alternatives and three benefit criteria for evaluating the alternatives, where $K=K_{i}=\left\{m_{1}, m_{2}, m_{3}\right\}$ and $K_{i i}=\emptyset$.

Table 2 Experts' opinion related to the cognitive development

\begin{tabular}{llll}
\hline & $m_{1}$ & $m_{2}$ & $m_{3}$ \\
\hline$c_{1}$ & $(0.69,0.54)$ & $(0.76,0.41)$ & $(0.87,0.27)$ \\
$c_{2}$ & $(0.73,0.48)$ & $(0.89,0.19)$ & $(0.56,0.59)$ \\
$c_{3}$ & $(0.84,0.34)$ & $(0.72,0.39)$ & $(0.61,0.44)$ \\
$f_{M}\left(m_{i}\right)$ & $(0.72,0.56)$ & $(0.75,0.63)$ & $(0.86,0.40)$ \\
\hline
\end{tabular}

Table 3 Experts' opinion related to the behavioral development

\begin{tabular}{llll}
\hline & $n_{1}$ & $n_{2}$ & $n_{3}$ \\
\hline$d_{1}$ & $(0.61,0.64)$ & $(0.66,0.51)$ & $(0.77,0.37)$ \\
$d_{2}$ & $(0.73,0.48)$ & $(0.95,0.09)$ & $(0.66,0.49)$ \\
$d_{3}$ & $(0.74,0.42)$ & $(0.62,0.49)$ & $(0.51,0.54)$ \\
$g_{N}\left(n_{i}\right)$ & $(0.65,0.51)$ & $(0.86,0.32)$ & $(0.79,0.49)$ \\
\hline
\end{tabular}


The values $p(+)_{j}$ and $p(-)_{j}$ for $(F, M, f)$ are calculated by using (4.2) and (4.3):

$p(+)_{j}=\{(0.84,0.34),(0.89,0.19),(0.87,0.27)\}$

$p(-)_{j}=\{(0.69,0.54),(0.72,0.41),(0.56,0.59)\}$

Using (4.9), the distance measures are computed for $\Omega_{H}\left(p(-)_{j}, p(+)_{j}\right)$ and $\Omega_{H}\left(p_{i j}, p(+)_{j}\right): \Omega_{H}\left(p(-)_{1}, p(+)_{1}\right)$ $=0.124, \Omega_{H}\left(p(-)_{2}, p(+)_{2}\right)=0.164, \Omega_{H}\left(p(-)_{3}, p(+)_{3}\right)$ $=0.18$.

$\Omega_{H}\left(p_{i j}, p(+)_{j}\right)=\left[\begin{array}{ccc}0.106 & 0.136 & 0 \\ 0.096 & 0 & 0.19 \\ 0 & 0.162 & 0.222\end{array}\right]$

The displaced RIs are calculated as:

$R I\left(p_{i j}\right)=\left[\begin{array}{ccc}0.855 & 0.83 & 0 \\ 0.774 & 0 & 1.056 \\ 0 & 0.99 & 1.23\end{array}\right]$

The PPFS values of the PF Importance weights are established by the expert group by evaluating the process: For $\omega_{j}=\left(\underline{\omega}_{j}, \bar{\omega}_{j}\right), \quad \omega_{1}=(0.8256,0.1935), \omega_{2}=(0.7756$, $0.2559), \omega_{3}=(0.7226,0.2234)$.

For compromise ranking of the alternatives, the values $\widetilde{\mathbb{S}}\left(c_{i}\right), \widetilde{\mathbb{R}}\left(c_{i}\right)$ and $\widetilde{\mathbb{Q}}\left(c_{i}\right)$ are as in Table 4 :

Now,

$\left.\left.\widetilde{\mathbb{Q}}^{(} c_{2}\right)-\widetilde{\mathbb{Q}}^{(} c_{1}\right)=0.49-0.222=0.268<\frac{1}{3-1}=0.5$. The first condition in $\mathbf{S ~} \mathbf{8}$ of Algorithm 1 is not satisfied. Then, the ultimate compromise solution is proposed. Therefore the order is $\left\{c_{1}, c_{2}\right\}<c_{3}$.

Algorithm 1 for $(F, N, g)$ :

Consider $K=K_{i}=\left\{n_{1}, n_{2}, n_{3}\right\}$ and $K_{i i}=\emptyset$. The values $p(+)_{j}$ and $p(-)_{j}$ :

$p(+)_{j}=\{(0.73,0.42),(0.95,0.09),(0.77,0.37)\}$

$p(-)_{j}=\{(0.61,0.64),(0.62,0.51),(0.51,0.54)\}$

The distance measures are computed as

$\Omega_{H}\left(p(-)_{1}, p(+)_{1}\right)=0.126, \Omega_{H}\left(p(-)_{2}, p(+)_{2}\right)=0.3162$,

$\Omega_{H}\left(p(-)_{3}, p(+)_{3}\right)=0.183$.

$\Omega_{H}\left(p_{i j}, p(+)_{j}\right)=\left[\begin{array}{ccc}0.1276 & 0.322 & 0 \\ 0.0364 & 0 & 0.06 \\ 0 & 0.3832 & 0.16\end{array}\right]$

The displaced RIs are calculated as:

$R I\left(p_{i j}\right)=\left[\begin{array}{ccc}1.013 & 1.018 & 0 \\ 0.289 & 0 & 0.33 \\ 0 & 1.212 & 0.874\end{array}\right]$
Table 4 Ranking with respect to the cognitive development for Algorithm 1

\begin{tabular}{lllll}
\hline & $m_{1}$ & $m_{2}$ & $m_{3}$ & Ranking \\
\hline$\widetilde{\mathbb{S}}\left(c_{i}\right)$ & 1.463 & 1.491 & 1.4175 & $c_{3}<c_{1}<c_{2}$ \\
$\widetilde{\mathbb{R}}\left(c_{i}\right)$ & 0.78 & 0.753 & 0.765 & $c_{1}<c_{3}<c_{2}$ \\
$\widetilde{\mathbb{Q}}\left(c_{i}\right)$ & 1 & 0.49 & 0.222 & $c_{1}<c_{2}<c_{3}$ \\
\hline
\end{tabular}

The PPFS values of the PF Importance weights: $\omega_{1}=$ $(0.7139,0.2821), \omega_{2}=(0.6983,0.3467), \omega_{3}=\quad(0.6762$, $0.3345)$.

For compromise ranking of the alternatives, the values $\widetilde{\mathbb{S}}\left(c_{i}\right), \widetilde{\mathbb{R}}\left(c_{i}\right)$ and $\widetilde{\mathbb{Q}}\left(c_{i}\right)$ are as in Table 5:

$\left.\left.\widetilde{\mathbb{Q}}^{(} d_{1}\right)-\widetilde{\mathbb{Q}}^{(} d_{2}\right)=0.313-0.000=0.313<\frac{1}{3-1}=0.5$.

The first condition in $\mathbf{S ~} \mathbf{8}$ of Algorithm 1 is not satisfied. Then, the ultimate compromise solution is proposed. Therefore the order is $\left\{d_{1}, d_{2}\right\}<d_{3}$.

Algorithm 2 for $(F, M, f)$ :

The values $\mathbb{S F}\left(c_{i}\right), \mathbb{R F}\left(c_{i}\right)$ and $\mathbb{Q} \mathbb{F}\left(c_{i}\right)$ as in Table 6:

$\mathbb{Q F}\left(c_{2}\right)-\mathbb{Q} \mathbb{F}\left(c_{1}\right)=0.58-0=0.58>\frac{1}{3-1}=0.5$.

Then, both of the conditions in $\mathbf{S} \mathbf{8}$ of Algorithm 2 are satisfied. Thus, the order is $c_{1}<c_{2}<c_{3}$.

Algorithm 2 for $(F, N, g)$ :

The values $\mathbb{S F}\left(d_{i}\right), \mathbb{R F}\left(d_{i}\right)$ and $\mathbb{Q} \mathbb{F}\left(d_{i}\right)$ as in Table 7:

$\mathbb{Q} \mathbb{F}\left(d_{2}\right)-\mathbb{Q} \mathbb{F}\left(d_{1}\right)=0.87-0=0.87>\frac{1}{3-1}=0.5$.

Then, both of the conditions in $\mathbf{S ~} \mathbf{8}$ of Algorithm 2 are satisfied. Thus, the order is $d_{2}<d_{1}<d_{3}$.

Table 5 Ranking with respect to the behavioral development for Algorithm 1

\begin{tabular}{lllll}
\hline & $n_{1}$ & $n_{2}$ & $n_{3}$ & Ranking \\
\hline$\widetilde{\mathbb{S}}\left(d_{i}\right)$ & 1.397 & 0.695 & 2.837 & $d_{2}<d_{1}<d_{3}$ \\
$\widetilde{\mathbb{R}}\left(d_{i}\right)$ & 0.71 & 0.35 & 1.491 & $d_{2}<d_{1}<d_{3}$ \\
$\widetilde{\mathbb{Q}}\left(d_{i}\right)$ & 0.313 & 0 & 1 & $d_{3}<d_{1}<d_{2}$
\end{tabular}

Table 6 Ranking with respect to the cognitive development for Algorithm 2

\begin{tabular}{lllll}
\hline & $m_{1}$ & $m_{2}$ & $m_{3}$ & Ranking \\
\hline $\mathbb{S F}\left(c_{i}\right)$ & 1.443 & 1.4765 & 1.5625 & $c_{1}<c_{2}<c_{3}$ \\
$\mathbb{R F}\left(c_{i}\right)$ & 0.573 & 0.5965 & 0.6 & $c_{1}<c_{2}<c_{3}$ \\
$\mathbb{Q} \mathbb{F}\left(c_{i}\right)$ & 0 & 0.58 & 1 & $c_{1}<c_{2}<c_{3}$ \\
\hline
\end{tabular}


Table 7 Ranking with respect to the behavioral development for Algorithm 2

\begin{tabular}{lllll}
\hline & $n_{1}$ & $n_{2}$ & $n_{3}$ & Ranking \\
\hline $\mathbb{S F}\left(d_{i}\right)$ & 1.5975 & 1.235 & 1.7265 & $d_{2}<d_{1}<d_{3}$ \\
$\mathbb{R} \mathbb{F}\left(d_{i}\right)$ & 0.6125 & 0.54 & 0.61 & $d_{1}<d_{3}<d_{2}$ \\
$\mathbb{Q F}\left(d_{i}\right)$ & 0.87 & 0 & 0.985 & $d_{2}<d_{1}<d_{3}$ \\
\hline
\end{tabular}

Table 8 Ranking with respect to the cognitive development for Algorithm 3

\begin{tabular}{lllll}
\hline & $m_{1}$ & $m_{2}$ & $m_{3}$ & Ranking \\
\hline$\widetilde{\mathbb{S}}\left(c_{i}\right)$ & 0.506 & 0.658 & 0.784 & $c_{1}<c_{2}<c_{3}$ \\
$\widetilde{\mathbb{R}}\left(c_{i}\right)$ & 0.261 & 0.422 & 0.492 & $c_{1}<c_{2}<c_{3}$ \\
$\widetilde{\mathbb{Q}}\left(c_{i}\right)$ & 0 & 0.6234 & 1 & $c_{1}<c_{2}<c_{3}$ \\
\hline
\end{tabular}

Table 9 Ranking with respect to the behavioral development for Algorithm 3

\begin{tabular}{lllll}
\hline & $n_{1}$ & $n_{2}$ & $n_{3}$ & Ranking \\
\hline$\widetilde{\mathbb{S}}\left(d_{i}\right)$ & 0.6815 & 0.913 & 0.764 & $d_{1}<d_{3}<d_{2}$ \\
$\widetilde{\mathbb{R}}\left(d_{i}\right)$ & 0.4 & 0.803 & 0.474 & $d_{1}<d_{3}<d_{2}$ \\
$\widetilde{\mathbb{Q}}\left(d_{i}\right)$ & 0 & 1 & 0.28 & $d_{1}<d_{3}<d_{2}$ \\
\hline
\end{tabular}

\section{Algorithm 3 for $(F, M, f)$ :}

The values $p(+)_{j}$ and $p(-)_{j}$ for $(F, M, f)$ as follows:

$$
\begin{aligned}
& p(+)_{j}=\{(0.84,0.34),(0.89,0.19),(0.87,0.27)\} \\
& p(-)_{j}=\{(0.69,0.54),(0.72,0.41),(0.56,0.59)\} .
\end{aligned}
$$

The distance measures are computed for $\Omega_{H}\left(p(-)_{j}, p(+)_{j}\right)$ and $\quad \Omega_{H}\left(p_{i j}, p(+)_{j}\right): \quad \Omega_{H}\left(p(-)_{1}, p(+)_{1}\right)=0.124$, $\Omega_{H}\left(p(-)_{2}, p(+)_{2}\right)=0.164, \Omega_{H}\left(p(-)_{3}, p(+)_{3}\right)=0.18$ and $\Omega_{H}\left(p_{11}, p(+)_{1}\right)=0.106, \Omega_{H}\left(p_{12}, p(+)_{2}\right)=0.136, \Omega_{H}\left(p_{13}\right.$, $\left.p(+)_{3}\right)=0, \Omega_{H}\left(p_{21}, p(+)_{1}\right)=0.096, \Omega_{H}\left(p_{22}, p(+)_{2}\right)=0$, $\Omega_{H}\left(p_{23}, p(+)_{3}\right)=0.19, \quad \Omega_{H}\left(p_{31}, p(+)_{1}\right)=0, \quad \Omega_{H}\left(p_{32}\right.$ ,$\left.p(+)_{2}\right)=0.162, \Omega_{H}\left(p_{33}, p(+)_{3}\right)=0.222$. Further, consider the RI values in (5.13).

Using the expectation score function, calculate the weights: $\mathscr{E} \mathscr{S}_{1}=0.6024, \mathscr{E} \mathscr{S}_{2}=0.5828, \mathscr{E} \mathscr{S}_{3}=0.7898$ and $\quad \omega_{1}=0.305012658, \quad \omega_{2}=0.295088608, \quad \omega_{3}=$ $0.399898734\left(\sum_{j=1}^{3} \omega_{j}=1\right)$. The RIs values are multiplied by precise weights $\omega_{j}$ :

$$
\begin{aligned}
& R I\left(p_{i j}\right) \cdot \omega_{j}=\left[\begin{array}{ccc}
0.261 & 0.245 & 0 \\
0.236 & 0 & 0.422 \\
0 & 0.292 & 0.492
\end{array}\right] \\
& \widetilde{\mathbb{Q}}\left(d_{2}\right)-\widetilde{\mathbb{Q}}\left(d_{1}\right)=0.6234-0=0.6234>\frac{1}{3-1}=0.5 .
\end{aligned}
$$

Then, both of the conditions in $\mathbf{S ~} \mathbf{8}$ of Algorithm 3 are satisfied. Thus, the order is $c_{1}<c_{2}<c_{3}$.

Algorithm 3 for $(F, N, g)$ :

$$
\begin{aligned}
& p(+)_{j}=\{(0.73,0.42),(0.95,0.09),(0.77,0.37)\} \\
& p(-)_{j}=\{(0.61,0.64),(0.62,0.51),(0.51,0.54)\}
\end{aligned}
$$

The distance measures are computed as $\Omega_{H}\left(p(-)_{1}, p(+)_{1}\right)=0.126, \quad \Omega_{H}\left(p(-)_{2}, p(+)_{2}\right)=0.3162$, $\Omega_{H}\left(p(-)_{3}, p(+)_{3}\right)=0.183$ and $\Omega_{H}\left(p_{11}, p(+)_{1}\right)=0.1276$, $\Omega_{H}\left(p_{12}, p(+)_{2}\right)=0.322, \quad \Omega_{H}\left(p_{13}, p(+)_{3}\right)=0, \quad \Omega_{H}\left(p_{21}\right.$, $\left.p(+)_{1}\right)=0.0364, \quad \Omega_{H}\left(p_{22}, p(+)_{2}\right)=0, \quad \Omega_{H}\left(p_{23}, p(+)_{3}\right)$ $=0.06, \quad \Omega_{H}\left(p_{31}, p(+)_{1}\right)=0, \quad \Omega_{H}\left(p_{32}, p(+)_{2}\right)=0.3832$, $\Omega_{H}\left(p_{33}, p(+)_{3}\right)=0.16$. Further, consider the RI values in (5.14).

Using the expectation score function, calculate the weights: $\mathscr{E} \mathscr{S}_{1}=0.5812, \quad \mathscr{E} \mathscr{S}_{2}=0.8186, \quad \mathscr{E} \mathscr{S}_{3}=0.692$ and $\quad \omega_{1}=0.27784683, \quad \omega_{2}=0.391337604, \quad \omega_{3}=$ $0.330815566\left(\sum_{j=1}^{3} \omega_{j}=1\right)$. The RIs values are multiplying by precise weights $\omega_{j}$ :

$R I\left(p_{i j}\right) \cdot \omega_{j}=\left[\begin{array}{ccc}0.2815 & 0.4 & 0 \\ 0.803 & 0 & 0.11 \\ 0 & 0.474 & 0.29\end{array}\right]$

$$
\widetilde{\mathbb{Q}}\left(d_{2}\right)-\widetilde{\mathbb{Q}}\left(d_{1}\right)=0.28-0=0.28<\frac{1}{3-1}=0.5 . \quad \text { Then, }
$$
both of the conditions in $\mathbf{S ~} \mathbf{8}$ of Algorithm 3 are not satisfied. Thus, the order is $\left\{d_{1}, d_{2}\right\}<d_{3}$.

Algorithm 4 for $(F, M, f)$ :

The values $\widetilde{\mathbb{S F}}\left(c_{i}\right), \widetilde{\mathbb{R F}}\left(c_{i}\right)$ and $\widetilde{\mathbb{Q F}}\left(c_{i}\right)$ are as in Table 10:

$$
\widetilde{\mathbb{Q F}}\left(c_{2}\right)-\widetilde{\mathbb{Q F}}\left(c_{1}\right)=1-0.17=0.83>\frac{1}{3-1}=0.5 .
$$

Then, both of the conditions in $\mathbf{S} \mathbf{8}$ of Algorithm 4 are satisfied. Thus, the order is $c_{1}<c_{3}<c_{2}$.

Algorithm 4 for $(F, N, g)$ :

The values $\widetilde{\mathbb{S F}}\left(d_{i}\right), \widetilde{\mathbb{R F}}\left(d_{i}\right)$ and $\widetilde{\mathbb{Q F}}\left(d_{i}\right)$ as in Table 11:

$$
\widetilde{\mathbb{Q F}}\left(d_{2}\right)-\widetilde{\mathbb{Q F}}\left(d_{1}\right)=0.242-0=0.242<\frac{1}{3-1}=0.5 \text {. }
$$

Then, both of the conditions in $\mathbf{S ~} 8$ of Algorithm 4 are not satisfied. Thus, the order is $\left\{d_{1}, d_{2}\right\}<d_{3}$.

It is obvious that the remoteness index-based GPFSSVIKOR algorithms are simple and easy to perform. Besides facilitating operation and computational efficiency, it has the ability to generate admissibly and reasonable outcomes for the final preference rankings. Moreover, the algorithmic process corresponding to the suggested methods can ensure 
Table 10 Ranking with respect to the behavioral development for Algorithm 4

\begin{tabular}{lllll}
\hline & $m_{1}$ & $m_{2}$ & $m_{3}$ & Ranking \\
\hline$\widetilde{\mathbb{S F}}\left(c_{i}\right)$ & 0.5 & 0.475 & 0.55 & $c_{2}<c_{1}<c_{3}$ \\
$\widetilde{\mathbb{R F}}\left(c_{i}\right)$ & 0.19 & 0.208 & 0.29 & $c_{1}<c_{2}<c_{3}$ \\
$\widetilde{\mathbb{Q} F}\left(c_{i}\right)$ & 0.17 & 1.04 & 1 & $c_{1}<c_{3}<c_{2}$ \\
\hline
\end{tabular}

Table 11 Ranking with respect to the behavioral development for Algorithm 4

\begin{tabular}{lllll}
\hline & $n_{1}$ & $n_{2}$ & $n_{3}$ & Ranking \\
\hline$\widetilde{\mathbb{S F}}\left(d_{i}\right)$ & 0.597 & 0.497 & 0.704 & $d_{2}<d_{1}<d_{3}$ \\
$\widetilde{\mathbb{R F}}\left(d_{i}\right)$ & 0.227 & 0.227 & 0.274 & $d_{1}=d_{2}<d_{3}$ \\
$\widetilde{\mathbb{Q F}}\left(d_{i}\right)$ & 0.242 & 0 & 1 & $d_{2}<d_{1}<d_{3}$ \\
\hline
\end{tabular}

flexibility to adapt to the particularities in real-world decision cases, such as different preference information of criterion significance and distinct points of reference from displaced and fixed ideal views. As a whole, the practicability and applicability of the developed methods in the GPFSS have been proved via real-world practice to the cognitive and behavioral development of early childhood children. Furthermore, as opposed to the available VIKOR approaches the suggested methods can sufficiently describe more uncertainty and ambiguity inherent in the subjective DM process by employing the GPFSS information.

\subsection{Stability analysis}

In the application for algorithms, $\lambda=1$ and $\delta=0.5$ were chosen. Solution results for $\lambda=1$ and $\delta=0.5$ are shown in Table 12. When the value of parameter $\delta$ decreases from 1 to 0 , the individual regret gets importance. The ranking of alternatives varying when the values of $\delta$ change, but the alternative $y 4$ remains the best option from alternatives. Also, different values of the parameter $\mathrm{p}$ are used for Algorithms 1-4. Algorithms 1 and 3 used the displaced terminologies and concepts, while Algorithms 2 and 4 are based on the fixed terminologies and concepts. Algorithms 1 and 3 are more sensitive as compared to Algorithms 2 and 4 , i.e., the ranking of the alternatives changes rapidly by changing the values of the parameter $\delta$. For more details, we refer to Tables 13 and 14 .

\subsection{Comparison with some methods}

If there are conflicting criteria among the alternatives, the sorting and selection procedures for these criteria can be carried out. These are the main features of the VIKOR method. In this method, majority and opponent describe maximum group utility and minimum individual regret, respectively. Providing these situations with the VIKOR approach helps decision-makers to obtain a compromise solution.

While the VIKOR method expresses the aggregation operator representing the closest to the ideal, TOPSIS determines a solution with the shortest distance to the ideal solution and the furthest distance to the negative ideal solution. Both methods consider that there exists a performance matrix obtained by the evaluation of all the alternatives in terms of each criterion. Normalization is used to eliminate the units of criterion values. The VIKOR method was created with the idea of nearness to the ideal and emerged from an aggregation operator using linear normalization. TOPSIS method uses vector normalization as normalization. This normalization does not take into account the relative importance of the distances from these points while using two reference points. The main difference appears in the aggregation approaches. An aggregating function is formulated, and it is used as a ranking index. In addition to ranking, the VIKOR method proposes a compromise solution with an advantage rate.

Ranking by PROMETHEE [9, 10], with a linear preference function, gives the same outcomes as ranking by VIKOR, with measures symbolizing group utility. The different weights are less important in the PROMETHEE method than in the VIKOR method. Rankings obtained using TOPSIS and PROMETHEE usual methods have a strong relationship between themselves. Although the PROMETHEE method does not use normalization, it gives
Table 12 Results of Solutions for $\lambda=1$ and $\delta=0.5$

\begin{tabular}{llll}
\hline Weights & Algorithm & $\begin{array}{l}\text { for }(F, M, f) \\
\text { Ranking }\end{array}$ & $\begin{array}{l}\text { for }(F, N, g) \\
\text { Ranking }\end{array}$ \\
\hline PF Importance weights & Algorithm 1 & $\left\{c_{1}, c_{2}\right\}<c_{3}$ & $\left\{d_{1}, d_{2}\right\}<d_{3}$ \\
PF Importance weights & Algorithm 2 & $c_{1}<c_{2}<c_{3}$ & $\left\{d_{1}, d_{2}\right\}<d_{3}$ \\
Precise Importance weights & Algorithm 3 & $c_{1}<c_{2}<c_{3}$ & $\left\{d_{1}, d_{2}\right\}<d_{3}$ \\
Precise Importance weights & Algorithm 4 & $c_{1}<c_{3}<c_{2}$ & $\left\{d_{1}, d_{2}\right\}<d_{3}$ \\
\hline
\end{tabular}


Neural Computing and Applications (2022) 34:1877-1903

1899

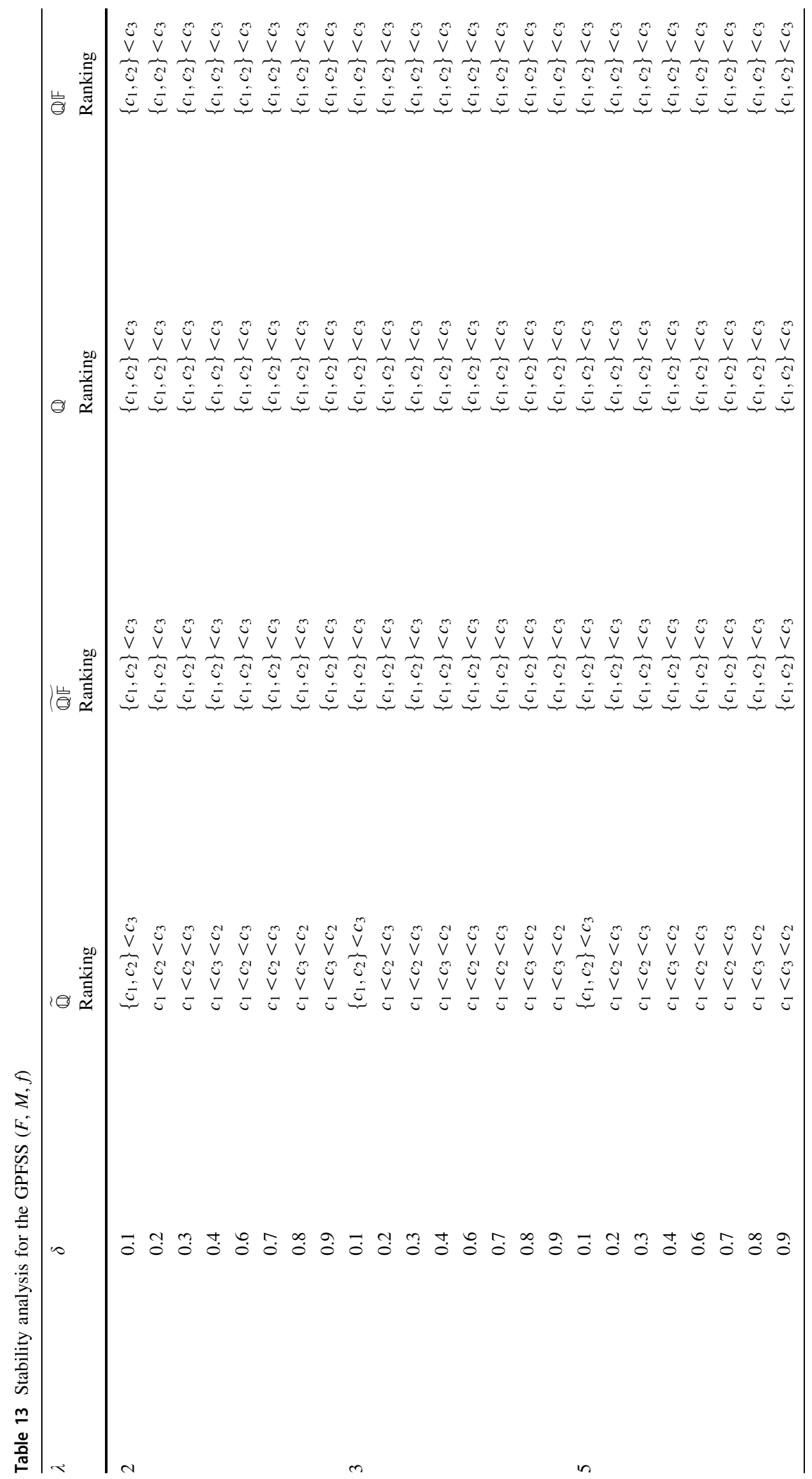

䈝 Springer 


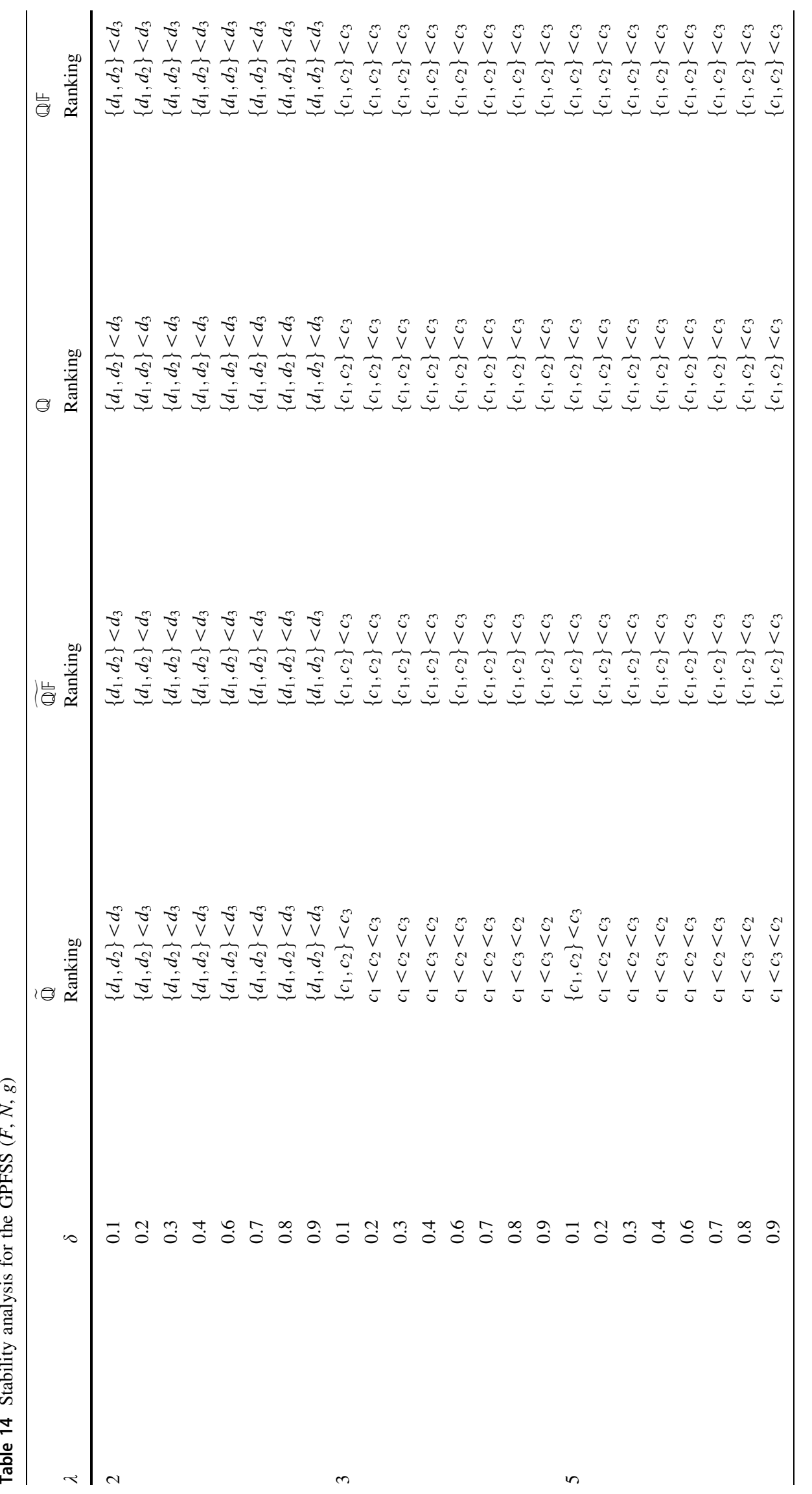


quite different results depending on the parameter set used. This indicates that method selection and configuration are important for the DM problem.

ELECTRE is an outranking method that is simple but provides partial ranking $[8,15]$. The ELECTRE method is the concept of elimination from a normalized result through a function that is further reduced to concordance and discordance to rank. The ELECTRE method has a basic understanding of the same data as a previously normalized VIKOR with rules. The largest value is the best value, or the smallest value is the best value. The end result can be searched by mathematical deduction of concordance and discordance resulting in a ranking of alternatives that can be decided.

VIKOR technique provides an effective DM method in domains in which the selection of the best alternative is a highly complex environment. Therefore, the VIKOR method may give better results than the DM methods discussed here.

\section{Discussion and conclusion}

The concept of GPFFS has defined by Kirisci [27]. This new definition adds the generalization parameter to the pool of PFNs and extends PFSS to GPFSS. The concept of GPFSS, which emerged from PFSS, functions to evaluate the information obtained with a parameter that reflects the views of an expert. It is important that the uncertainty associated with an observer be verified by an expert in the choice of degree of membership, and this is the main motivation for developing the GPFSS concept. The Pythagorean fuzzy soft sets are generalized by introducing the generalization parameter given by an expert to validate the original Pythagorean fuzzy values. The role of the expert is to refine PFNs with his domain-specific knowledge. It may be noted that information of any sort often gets misinterpreted during its presentation. This usually happens as the presenter has no domain-specific knowledge or lacks the standard terminologies, and this is sought to be corrected by the proposed GPFSS. An expert can be viewed as a domain professional having years of experience. The expert's opinion indicates the credibility of the evaluation of the alternative by the experts. This opinion coming from the expert constitutes the generalization parameter. The severity of symptoms as reported by the patients in the linguistic form as membership grades can be used in the PFSS.

In this study, new Euclidean, Hamming, and generalized distance measures were given for GPFSSs according to the new PFNs $P=\left(r_{P}, d_{P}\right)$, and their basic properties were examined. Pythagorean membership grade was defined by Yager [45] as a new pair of PFNs $\left(r_{P}, d_{P}\right)$. Here, $r_{P}$ is stated as the strength of commitment and $d_{P}$ as the direction of commitment. $r_{p}$ and $d_{p}$ are characterized by $u_{p}=r_{p} \cos \left(\theta_{p}\right), v_{p}=r_{p} \sin \left(\theta_{p}\right)$. These values are related to MS and NMS grades. The new distance measures have been used to build useful concepts of remoteness indices, consisting of displaced positive- and negative-ideal remoteness indices as well as fixed positive- and negativeideal remoteness indices. After distance measurements, new remoteness index-based GPFSS-VIKOR methods have been developed to address MCDA problems in the GPFSS environment, using the concepts of displaced and fixed remoteness indices based on new distance measures. These VIKOR methods are a generalization of the standard VIKOR. This generalization is a new compromising decision-making method based on the proposed generalized distance measure and the displaced and fixed remoteness index. In the DM process, appropriate reference points need to be determined to facilitate the definition of displaced and fixed remoteness indices because these points can effectively form anchored judgments in subjective decision-making processes. Finding these points is the first step for remoteness index-based GPFSS-VIKOR. Then, the displaced and fixed Remoteness Indexes were built. The displaced RI and fixed RI were defined, proved the properties, and given the relevant examples. It has been seen from the examples that the contrasts between the remoteness indices become less noticeable when anchoring subjective judgments with fixed ideal PF values. However, for the displaced ideal PF values, this situation is not very clear.

In decision-making processes, the criteria that determine the decisions are not of equal importance. Therefore, the criteria are weighted when making a decision. These weights are used to determine alternatives and make choices according to the values of subjective evaluations. Two types of weights were used in this study. The first of these weights is calculated with the expectation score function. The second is the weights obtained as a result of recording the evaluations of the experts as PFSS.

In this study, a multi-criteria ranking was created with remoteness indices based on displaced and fixed ideal PF values. Thus, remoteness-based VIKOR methods were built. The smaller the displaced remoteness index is, the more prior the evaluative rating is. In the new VIKOR methods, the priority relationship between the alternatives is evaluated on the basis of criteria. Thus, this priority relationship is defined in ascending order according to each displaced remoteness index because the placed ideal PF values have properties that facilitate anchored judgments. Since a compromise ranking is expected on all criteria, new definitions of a group utility index and an individual regret index are given to synthesize criterion-based priority 
relationships. As it can be understood from these explanations, in this study, the basic structure of VIKOR has been expanded and a new compromising decision-making method has been established with the proposed distance measures, the displaced remoteness index, and the fixed remoteness index. The method proposed by the study can be easily used to solve MCDA problems where data describing the performance of alternatives are characterized by $P F$ values.

In this study, a new way is proposed to evaluate an aggregated result for each alternative and to obtain a compromise solution. The remoteness indexes obtained based on the new distance measures have the function of formulating the remoteness based on multiple criteria ranking. The computational processes of the four algorithms presented here suggest various preference structures and are capable of fully exploiting the complex information contained in the PF decision matrix. Considering the VIKOR methods in the PF environment, the remotenessbased VIKOR methods proposed in this study are extremely flexible since they detect the location of the displaced and fixed reference points. These new VIKOR methods can be easily applied to real-life problems and solve many challenging decision-making problems. As seen in the reallife example given here, new remoteness-based VIKOR methods can be easily used in very difficult processes such as monitoring and evaluating cognitive and behavioral development. The target audience of the sample discussed is children aged 5-6 years. The effect of this age group's mandatory stay at home in COVID-19 quarantines on their development has been examined. The factors affecting the developmental processes of children aged 5-6 years were evaluated with the newly given remoteness-based VIKOR methods, and the factors affecting development were listed.

Acknowledgements We would like to thank the anonymous reviewers for their comments that helped us improve this manuscript.

Funding This research did not receive any specific grant from funding agencies in the public, commercial, or not-for-profit sectors.

\section{Declarations}

Conflict of interest The authors declare that they have no conflict of interest.

\section{References}

1. Agarwal M, Biswas KK, Hanmandlu M (2013) Generalized intuitionistic fuzzy sets with applications in decision making. Appl Soft Compt 13:3552-3566

2. Ali MA, Feng F, Liu X, Min WK, Shabir M (2009) On some new operations in soft set theory. Comput Math Appl 57:1547-1553

3. Atanassov K (1986) Intuitionistic fuzzy sets. Fuzzy Sets Syst 20:87-96
4. Athira TM, John SJ, Garg H (2019) Entropy and distance measures of Pythagorean fuzzy soft sets and their applications. J Intelli Fuzzy Syst 37(3):4071-4084. https://doi.org/10.3233/JIFS190217

5. Athira TM, John SJ, Garg H (2020) A novel entropy measure of Pythagorean fuzzy soft sets. AIMS Math 5(2):1050-1061. https:// doi.org/10.3934/math.2020073

6. Bagheri M, Shojaei P, Tayebi Khorami M (2018) A comparative survey of the condition of tourism infrastructure in Iranian provinces using VIKOR and TOPSIS. Decis Sci Lett 7(1):87-102

7. Banaeian N, Mobli H, Fahimnia B, Nielsen IE, Omid M (2018) Green supplier selection using fuzzy group decision making methods: a case study from the agri-food industry. Comput Oper Res 89:337-347

8. Benayoun R, Roy B, Sussman B. (1966) Une méthode pour guider le choix en presence de points de vue multiples. Note de travail 49. SEMA-METRA. Direction-Scientifique

9. Brans JP, Vincke PH (1985) A preference ranking organisation method (the PROMETHEE method for multiple criteria decisionmaking). Manag Sci 31(6):647-656

10. Brans JP, Mareschal B (2005) PROMETHEE Methods. State of the Art Surveys, Springer, New York, Multiple Criteria Decision Analysis, pp 163-195

11. Chatterjee P, Chakraborty S (2016). A comparative analysis of VIKOR method and its variants. Decis Sci Lett 5(4), 469-486

12. Chen TY (2018) Remoteness index-based Pythagorean fuzzy VIKOR methods with a generalized distance measure for multiple criteria decision analysis. Inf Fusion 41:129-150

13. Deli I, Cagman N (2015) Intuitionistic fuzzy parameterized soft set theory and its decision making. Appl Soft Comp 28:109-113

14. Feng F, Fujita H, Ali MA, Yager RR, Liu X, Another view on generalized intuitionistic fuzzy soft sets and related multi- attribute decision making methods. IEEE Trans Fuzzy Syst. https:// doi.org/10.1109/TFUZZ.2018.2860967

15. Figueira JR, Greco S, Roy B, Słowiński R (2013) An overview of ELECTRE methods and their recent extensions. J Multi-Criteria Decis Anal 20:61-85

16. Garg H (2016) A new generalized Pythagorean fuzzy information aggregation using Einstein operations and its application to decision making. Int J Intell Syst 31:886-920

17. Garg H (2016) A novel correlation coefficients between Pythagorean fuzzy sets and its applications to decision-making process. Int J Intell Syst 31(12):1234-1252. https://doi.org/10.1002/int. 21827

18. Garg H (2016) Some series of intuitionsitic averaging aggregation operators. SpringerPlus 5(1):999. https://doi.org/10.1186/ s40064-016-2591-9

19. Garg H (2016) A new generalized improved score function of interval-valued intuitionistic fuzzy sets and applications in expert systems. Appl Soft Comput 38:988-999. https://doi.org/10.1016/ j.asoc.2015.10.040

20. Garg H, Arora R (2017) Distance and similarity measures for dual hesitant fuzzy soft sets and their applications in multicriteria decision-making problem. Int J Uncertain Quant 7(3):229-229. https://doi.org/10.1615/Int.J.Uncertainty (2017019801)

21. Gul M, Celik E, Aydin N, Taskin Gumus A, Guneri AF (2016) A state of the art literature review of VIKOR and its fuzzy extensions on applications. Appl Soft Comput 46:60-89

22. Guleria A, Bajaj RK (2018) On Pythagorean fuzzy matrices, operations and their applications in decision making and medical diagnosis. Soft Comput 23:7889. https://doi.org/10.1007/s00500$018-3419-\mathrm{z}$

23. Kirişci M (2019) Comparison the medical decision-making with Intuitionistic fuzzy parameterized fuzzy soft set and Riesz Summability. New Math Natural Comput 15:351-359. https:// doi.org/10.1142/S1793005719500194 
24. Kirisci M, Yilmaz H, Saka MU (2019) An ANFIS perspective for the diagnosis of type II diabetes. Ann Fuzzy Math Inform 17:101-113

25. Kirişci M (2020) Medical decision making with respect to the fuzzy soft sets. J Interdiscip Math 23:767-776. https://doi.org/10. $1080 / 09720502.2020 .1715577$

26. Kirişci M (2020) A case study for medical decision making with the fuzzy soft sets. Afrika Matematika 31:557-564. https://doi. org/10.1007/s13370-019-00741-9

27. Kirişci M (2021) $\Omega$ - sodt sets and medical decision-making application. Int J Comput Math. https://doi.org/10.1080/ 00207160.2020 .1777404

28. Liao H, Xu Z (2013) A VIKOR-based method for hesitant fuzzy multi-criteria decision making. Fuzzy Optim Decis Mak 12(4):373-392

29. Maji PK, Biswas R, Roy AR (2001) Fuzzy soft sets. J Fuzzy Math 9:589-602

30. Maji PK, Biswas R, Roy AR (2001) Intuitionistic fuzzy soft sets. Comput Math Appl 9:677-692

31. Maji PK, Biswas R, Roy AR (2003) Soft set theory. Comp Math Appl 45:555-562

32. Majumdar P, Samanta S (2008) Similarity measure of soft sets. New Math Natural Comput 4(1):1-12

33. Molodtsov D (1999) Soft set theory-first results. Comput Math Appl 20:239-244

34. Naeem K, Riaz M, Peng X, Afzal D (2019) Pythagorean fuzzy soft MCGDM methods based on TOPSIS, VIKOR and aggregation operators. J Intell Fuzzy Syst 37(5):6937-6957. https://doi. org/10.3233/JIFS-190905

35. Opricovic S, Tzeng GH (2004) Compromise solution by MCDM methods: a comparative analysis of VIKOR and TOPSIS. Eur J Op Res 156(2):445-455

36. Opricovic S, Tzeng GH (2002) Multicriteria planning of postearthquake sustainable reconstruction. Comput Aided Civi Infrastruct Eng 17(3):211-220

37. Opricovic S, Tzeng G-H (2007) Extended VIKOR method in comparison with outranking methods. Eur J Oper Res 178(2):514-529

38. Peng X, Yang Y, Song J, Jiang Y (2015) Pythagorean fuzzy soft set and its application. Comput Eng 41:224-229
39. Peng X, Yang Y (2015) Some results for Pythagorean fuzzy sets. Int J Intell Syst 30:1133-1160

40. Peng X, Yang Y (2016) Multiple attribute group decision making methods based on Pythagorean fuzzy linguistic set. Comput Eng Appl 52(3):50-54

41. Peng X, Selvachandran G (2019) Pythagorean fuzzy set: state of the art and future directions. Artif Intell Rev 52:1873-1927. https://doi.org/10.1007/s10462-017-9596-9

42. Shahzadi G, Akram A (2019) Hypergraphs based on Pythagorean fuzzy soft model. Math Comput Appl 24:100. https://doi.org/10. $3390 /$ mca24040100

43. Smarandache F (2005) Neutrosophic set, a generalisation of the intuitionistic fuzzy sets. Inter J Pure Appl Math 24:287-297

44. Xu ZS (2007) Intuitionistic fuzzy aggregation operator. IEEE Trans Fuzzy Syst 15:1179-1187. https://doi.org/10.1109/TFUZZ. 2006.890678

45. Yager RR (2013) Pythagorean fuzzy subsets. Proc Joint IFSA World Congress and NAFIPS Annual Meeting, Edmonton, Canada

46. Yager RR (2014) Pythagorean membership grade in multicriteria decision making. IEEE Fuzzy Syst 22:958-965

47. Yager RR (2016) Multicriteria decision making with ordinal linguistic intuitionistic fuzzy sets for mobile apps. IEEE Trans Fuzzy Syst 24:590-599

48. Yager RR, Abbasov AM (2013) Pythagorean membership grades, complex numbers and decision making. Int $\mathbf{J}$ Intell Syst 28:436-452

49. Zadeh LA (1965) Fuzzy sets. Inf Comp 8:338-353

50. Zeng Q-L, Li D-D, Yang Y-B (2013) VIKOR method with enhanced accuracy for multiple criteria decision making in healthcare management. J Med Syst 37:1-9

51. Zhang XL, Xu ZS (2014) Extension of TOPSIS to multi-criteria decision making with Pythagorean fuzzy sets. Int J Intell Syst 29:1061-1078

Publisher's Note Springer Nature remains neutral with regard to jurisdictional claims in published maps and institutional affiliations. 